\title{
MEGARA, the $R=6000-20000$ IFU and MOS of GTC
}

\section{Carrasco, E., Gil de Paz, A., Gallego, J., Iglesias-Páramo, J., Cedazo, R., et al.}

E. Carrasco, A. Gil de Paz, J. Gallego, J. Iglesias-Páramo, R. Cedazo, M. L. García Vargas, X. Arrillaga, J. L. Avilés, A. Bouquin, J. Carbajo, N. Cardiel, M. A. Carrera, A. Castillo Morales, E. Castillo-Domínguez, S. Esteban San Román, D. Ferrusca, P. Gómez-Álvarez, R. IzazagaPérez, B. Lefort, J. A. López Orozco, M. Maldonado, I. Martínez Delgado, I. Morales Durán, E. Mújica, R. Ortiz, G. Páez, S. Pascual, A. Pérez-Calpena, P. Picazo, A. Sánchez-Penim, E. Sánchez-Blanco, S. Tulloch, M. Velázquez, J. M. Vílchez, J. Zamorano, A. L. Aguerri, D. Barrado, E. Bertone, A. Cava, C. Catalán-Torrecilla, J. Cenarro, M. Chávez, B. T. Dullo, C. Eliche, Mi. García, J. García-Rojas, J. Guichard, R. González-Delgado, R. Guzmán, A. Herrero, N. Huélamo, D. H. Hughes, J. Jiménez-Vicente, C. Kehrig, R. A. Marino, I. Márquez, J. Masegosa, D. Mayya, J. Méndez-Abreu, M. Mollá, C. Muñoz-Tuñón, M. Peimbert, P. G. Pérez-González, E. Pérez-Montero, S. Roca-Fàbrega, M. Rodríguez, J. M. RodríguezEspinosa, L. Rodríguez-Merino, L. Rodríguez-Muñoz, D. Rosa-González, J. SánchezAlmeida, C. Sánchez Contreras, P. Sánchez-Blázquez, S. F. Sánchez, A. Sarajedini, S. Silich, S. Simón-Díaz, G. Tenorio-Tagle, E. Terlevich, R. Terlevich, S. Torres-Peimbert, I. Trujillo, Y. Tsamis, O. Vega, "MEGARA, the $R=6000-20000$ IFU and MOS of GTC," Proc. SPIE 10702, Ground-based and Airborne Instrumentation for Astronomy VII, 1070216 (6 July 2018); doi: $10.1117 / 12.2313040$

Event: SPIE Astronomical Telescopes + Instrumentation, 2018, Austin, Texas, United States 


\title{
MEGARA, the R=6000-20000 IFU and MOS of GTC
}

E. Carrasco $^{* a}$, A. Gil de Paz ${ }^{\mathrm{b}}$, J. Gallego ${ }^{\mathrm{b}}, \mathrm{J}_{\text {Iglesias-Páramo }}^{\mathrm{c}}$, R. Cedazo ${ }^{\mathrm{d}}$, M.L. García Vargas ${ }^{\mathrm{e}}$, X.

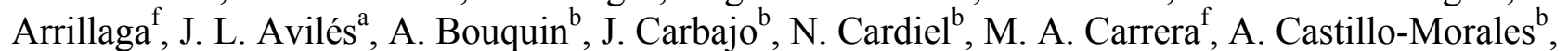

E. Castillo-Domínguez ${ }^{c}, S$. Esteban San Román ${ }^{b}$, D. Ferrusca ${ }^{a}$, P. Gómez-Álvarez ${ }^{\mathrm{e}}$, R. Izazaga-

Pérez $^{\mathrm{a}}$, B. Lefort ${ }^{\mathrm{e}}$, J.A. López-Orozco ${ }^{\mathrm{b}}$, M. Maldonado ${ }^{\mathrm{e}}$, I. Martínez-Delgado ${ }^{\mathrm{e}}, \mathrm{I}_{\text {. Morales Durán }}^{\mathrm{c}}$, E. Mujica $^{\mathrm{e}}$, R. Ortiz', G. Páez ${ }^{\mathrm{g}}$, S. Pascual ${ }^{\mathrm{b}}$, A. Pérez-Calpena ${ }^{\mathrm{e}}$, P. Picazo ${ }^{\mathrm{b}}$, A. Sánchez-Penim ${ }^{\mathrm{b}}$, E.

Sánchez-Blanco $^{\mathrm{e}}$, S. Tulloch ${ }^{\mathrm{e}}$, M. Velázquez ${ }^{\mathrm{b}}$, J.M. Vílchez $^{\mathrm{c}}$, J. Zamorano ${ }^{\mathrm{b}}$, A.L. Aguerri,i, D.

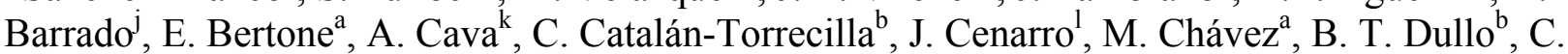

Eliche $^{\mathrm{h}}$, M. García ${ }^{\mathrm{j}}$, J. García-Rojas ${ }^{\mathrm{h}, \mathrm{i}}$, J. Guichard ${ }^{\mathrm{a}}$, R. González-Delgado ${ }^{\mathrm{c}}$, R. Gúzman ${ }^{\mathrm{m}}, \mathrm{A}$.

Herrero $^{\text {h,i }}$, N. Huélamo, D. H. Hughes ${ }^{\mathrm{a}}$, J. Jiménez-Vicente ${ }^{\mathrm{n}}$, C. Kehrig ${ }^{\mathrm{c}}$, R.A. Marino ${ }^{\mathrm{o}}$, I. Márquez ${ }^{\mathrm{c}}$,

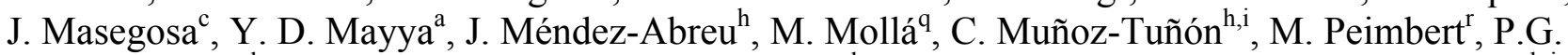
Pérez-González ${ }^{\mathrm{b}}$, E. Pérez Montero ${ }^{\mathrm{c}}$, S. Roca-Fàbrega ${ }^{\mathrm{b}}$, M. Rodríguez ${ }^{\mathrm{a}}$, J.M. Rodríguez-Espinosa ${ }^{\mathrm{h}, \mathrm{i}}$, L. Rodríguez-Merino ${ }^{\mathrm{a}}$, L. Rodríguez Muñoz ${ }^{\mathrm{s}}$ D. Rosa-González a , J. Sánchez-Almeida,i, C. Sánchez Contreras $^{j}$, P. Sánchez-Blázquez ${ }^{\mathrm{t}}$, S. F. Sánchez ${ }^{\mathrm{r}}$, A. Sarajedinim ${ }^{\mathrm{m}}$, S. Silich ${ }^{\mathrm{a}}$, S. Simón-Díaz,i , G. Tenorio-Tagle $^{\mathrm{a}}$, E. Terlevich ${ }^{\mathrm{a}}$, R. Terlevich ${ }^{\mathrm{a}}, \mathrm{S}_{\text {. Torres-Peimbert }}{ }^{\mathrm{r}}$, I. Trujillo $^{\mathrm{h}, \mathrm{i}}$, Y. Tsamis $^{\mathrm{u}}$, O. Vega $^{\mathrm{a}}$

${ }^{a}$ Instituto Nacional de Astrofísica, Óptica y Electrónica (INAOE, Mexico), ${ }^{b}$ Universidad Complutense de Madrid (UCM, Spain), ${ }^{c}$ Instituto de Astrofísica de Andalucía (IAA-CSIC, Spain), ${ }^{\mathrm{d}}$ Universidad Politécnica de Madrid (UPM, Spain), ${ }^{\mathrm{e}}$ FRACTAL SLNE (Spain), ${ }^{\mathrm{f}}$ AVS SL, ${ }_{\mathrm{g}}$ Centro de Investigaciones en Óptica (Mexico), ${ }^{\mathrm{h}}$ Instituto de Astrofísica de Canarias (Spain), ${ }^{\mathrm{i}}$ Universidad de La Laguna (Spain), ${ }^{j}$ Centro de Astrobiología (CSIC-INTA, Spain), ${ }^{k}$ University of Geneva (CH),

${ }^{l}$ Centro de Estudios de Física del Cosmos de Aragón (Spain), ${ }^{\mathrm{m}}$ University of Florida (USA), ${ }^{\mathrm{n}}$ Universidad de Granada (Spain), ${ }^{\circ}$ Department of Physics, ETH Zurich (Switzerland), ${ }^{\mathrm{p}}$ University of St. Andrews, ${ }^{\mathrm{q}}$ CIEMAT (Spain), ${ }^{\mathrm{r}}$ Universidad Autónoma de México (Mexico), ${ }^{\mathrm{s}}$ Osservatorio Astronomico di Padova (Italy), ${ }^{t}$ Universidad Autónoma de Madrid (Spain), ${ }^{\mathrm{u} D e p a r t m e n t ~ o f ~ P h y s i c s ~}$ and Astronomy, University College London (UK)

\begin{abstract}
MEGARA is the new generation IFU and MOS optical spectrograph built for the $10.4 \mathrm{~m}$ Gran Telescopio CANARIAS (GTC). The project was developed by a consortium led by UCM (Spain) that also includes INAOE (Mexico), IAA-CSIC (Spain) and UPM (Spain). The instrument arrived to GTC on March $28^{\text {th }} 2017$ and was successfully integrated and commissioned at the telescope from May to August 2017. During the on-sky commissioning we demonstrated that MEGARA is a powerful and robust instrument that provides on-sky intermediate-to-high spectral resolutions $\mathrm{R}_{\mathrm{FWHM}} \sim$ $6,000,12,000$ and 20,000 at an unprecedented efficiency for these resolving powers in both its IFU and MOS modes. The IFU covers $12.5 \times 11.3 \operatorname{arcsec}^{2}$ while the MOS mode allows observing up to 92 objects in a region of $3.5 \times 3.5$ $\operatorname{arcmin}^{2}$. In this paper we describe the instrument main subsystems, including the Folded-Cassegrain unit, the fiber link, the spectrograph, the cryostat, the detector and the control subsystems, and its performance numbers obtained during commissioning where the fulfillment of the instrument requirements is demonstrated.
\end{abstract}

Keywords: Instrumentation: spectrographs, Techniques: spectroscopic

*bec@inaoep.mx phone +52 2222663100

Ground-based and Airborne Instrumentation for Astronomy VII, edited by Christopher J. Evans, Luc Simard, Hideki Takami, Proc. of SPIE Vol. 10702, 1070216 - (c) 2018 SPIE · CCC code: 0277-786X/18/\$18 · doi: 10.1117/12.2313040 


\section{INTRODUCTION}

\subsection{Background}

MEGARA is a versatile Integral Field Unit and Multi-Object Spectrograph that is now part of the suite of facility instruments of the $10.4 \mathrm{~m}$ GTC and fills the niche of intermediate-to-high spectral resolution spectroscopy at optical wavelengths. In this paper we describe the main characteristics of the instrument and the results of its commissioning, which took place on the period from May $8^{\text {th }}$ to August $31^{\text {st }} 2017$. Here we provide a brief summary to the historical background that led to the instrument as it is now under scientific exploitation by the GTC community, in terms of its characteristics and performance.

The initiative to design and build MEGARA started in response to a call for "Letters of Intent" issued by GRANTECAN S.A. (the Spanish public company responsible for the operation and maintenance of GTC) on March $4^{\text {th }} 2009$. This first call was followed by an "Announcement of Opportunity" issued on September $14^{\text {th }} 2009$ that led to a competitive Conceptual Design that was reviewed by an international panel of experts on July 2010. This endeavor was undertaken from the beginning by a Consortium of institutions led by the Universidad Complutense de Madrid (UCM, Spain) that also included the Instituto de Astrofísica, Óptica y Electrónica (INAOE, Mexico), the Instituto de Astrofísica de Andalucía (IAA-CSIC, Granada, Spain) and the Universidad Politécnica de Madrid (UPM, Spain). Major private contractors participating in the project from this early phase to its completion were Fractal S.L.N.E. and AVS.

From that moment to the signature of both the Detailed Design (April $28^{\text {th }}$ 2014) and Construction (May $5^{\text {th }}$ 2014) contracts almost four years passed with only the funding for the Preliminary Design Review (held in Madrid, Spain, on March 2012) being released to the UCM and the rest of the MEGARA Consortium. The cost overrun generated by the delay in releasing these funds was charged to the Guaranteed Time account of the MEGARA Consortium, which was reduced from the initially advertised 660 hours to 301,28 hours. In spite of the difficulties faced by the Consortium and its contractors, we together completed the Detailed Design, Construction and AIV phases in less than three years, delivering the instrument to GTC at the Roque de los Muchachos observatory (La Palma, Spain) on March $28^{\text {th }} 2017$.

\subsection{Main characteristics}

The MEGARA instrument offers GTC with both IFU and MOS capabilities at spectral resolutions $\mathrm{R}_{\mathrm{FWHM}} \sim 6000,12000$ and 20000 through the entire optical window from 365 to $970 \mathrm{~nm}$. The IFU, also called the Large Compact Bundle (LCB), covers a Field of View (FoV) of $12.5 \times 11.3 \mathrm{arcsec}^{2}$ using a total of 567 optical fibers for a spaxel size of 0.62 arcsec. This value comes from the use of $100 \mu \mathrm{m}$-core optical fibers that are fed by a $\mathrm{F} / 3$ beam that is converted from the F/17 focal ratio of the GTC foci using hexagonally shaped and packed microlenses. The IFU fibers are complemented by 56 fibers coming from 8 sky bundles placed in the outer edge of the flat, non-vignetted Folded-Cass FoV. All 623 fibers are then mounted on the LCB pseudo-slit at the spectrograph entrance focal plane. In addition, a total of 92 mini-IFUs of 7 fibers (i.e. 644 fibers identical to the ones used for the LCB) are mounted on the same number of robotic positioners that move in overlapping patrol areas that all together fully cover the $3.5 \times 3.5 \operatorname{arcmin}^{2}$ FoV around the central IFU. These 644 fibers are arranged in a dedicated pseudo-slit that can be automatically interchanged with the LCB pseudo-slit.

Table 1. MEGARA Main Characteristics.

\begin{tabular}{|l|l|}
\hline IFU (LCB) Field of View & $12.5 \times 11.3 \mathrm{arcsec}^{2}$ \\
\hline MOS Field of View & $92 \times 1.6$ arcsec diameter mini-IFUs in $3.5 \times 3.5 \operatorname{arcmin}^{2}$ \\
\hline Spaxel size for both LCB \& MOS & $0.62 \operatorname{arcsec}($ diameter of the circle inscribing the hexagon) \\
\hline Wavelength range & $365-970 \mathrm{~nm}$ \\
\hline Spectral resolution ( $\left.\mathrm{R}_{\mathrm{FWHM}}\right)$ & $\begin{array}{l}6,000 \text { (LR VPHs); 12,000 (MR VPHs); 20,000 (HR } \\
\text { VPHs; measured) }\end{array}$ \\
\hline Multiplexing LCB & 567 IFU fibers +56 (7 fiber x 8 bundles) sky fibers \\
\hline Multiplexing MOS & 644 fibers (7 fiber x 92 bundles) \\
\hline GTC foci & Folded-Cass F (Fiber-MOS) + Nasmyth A (spectrograph) \\
\hline
\end{tabular}


The fiber link is therefore composed by the 1267 fibers constituting the two instrument modes with a length of 44.5 meters each fiber and connects the so-called Fiber-MOS unit in the Folded-Cassegrain F focus of GTC, where the IFU and the MOS robotic positioners are located, to the corresponding pseudo-slit situated at the entrance focal-plane of the MEGARA spectrograph in the Nasmyth A platform (see Figure 1).

The pseudo-slits feed a F/3 collimator that generates a pupil with a free diameter of $160 \mathrm{~mm}$. The F/1.5 camera, which is placed at a fixed angle relative to the collimator of 68 degrees, focus the output beam on an e2V CCD231-84 CCD detector of 4096 x $411215 \mu \mathrm{m}$ pixels. The CCD is hosted in a liquid-Nitrogen open-cycle cryostat designed and constructed by the INAOE in Mexico and, at the time of writing these paper, has been continuously working at nominal pressure and temperature values for over a year.

Three different types of Volume Phase Holographic (VPH) gratings are available for use at the pupil of the MEGARA spectrograph: namely the Low-Resolution (LR) ones, where the hologram gelatin is sandwiched between two flat windows only (inclined 34 degrees relative to both collimator and camera) yielding $\mathrm{R} \sim 6000$ resolving powers, the Medium-Resolution (MR) ones with $R \sim 12000$, and High-Resolution (HR) ones with R 20000 (requirement was $\mathrm{R} \sim 18,700$ ). These last two sets of VPHs use prisms glued on each side of the flat windows to reach their spectral resolutions. The number of disperser elements built (6 LRs, $10 \mathrm{MRs}, 2 \mathrm{HRs}$ ) allow to cover the entire optical window at $\mathrm{R} \sim 6000$ and 12000 and the $\mathrm{H} \alpha$ and $\mathrm{CaT}$ spectral regions at $\mathrm{R}=20000$, although any other bluer wavelengths can be accomodated by design. Out of these 18 VPHs, 11 can be mounted simultaneously on the spectrograph rotating wheel (the so-called VPH wheel) and interchanged automatically in $\sim 1$ minute of time using a dedicated insertion mechanism.

The main characteristics of the MEGARA instrument are summarized in Table 1.

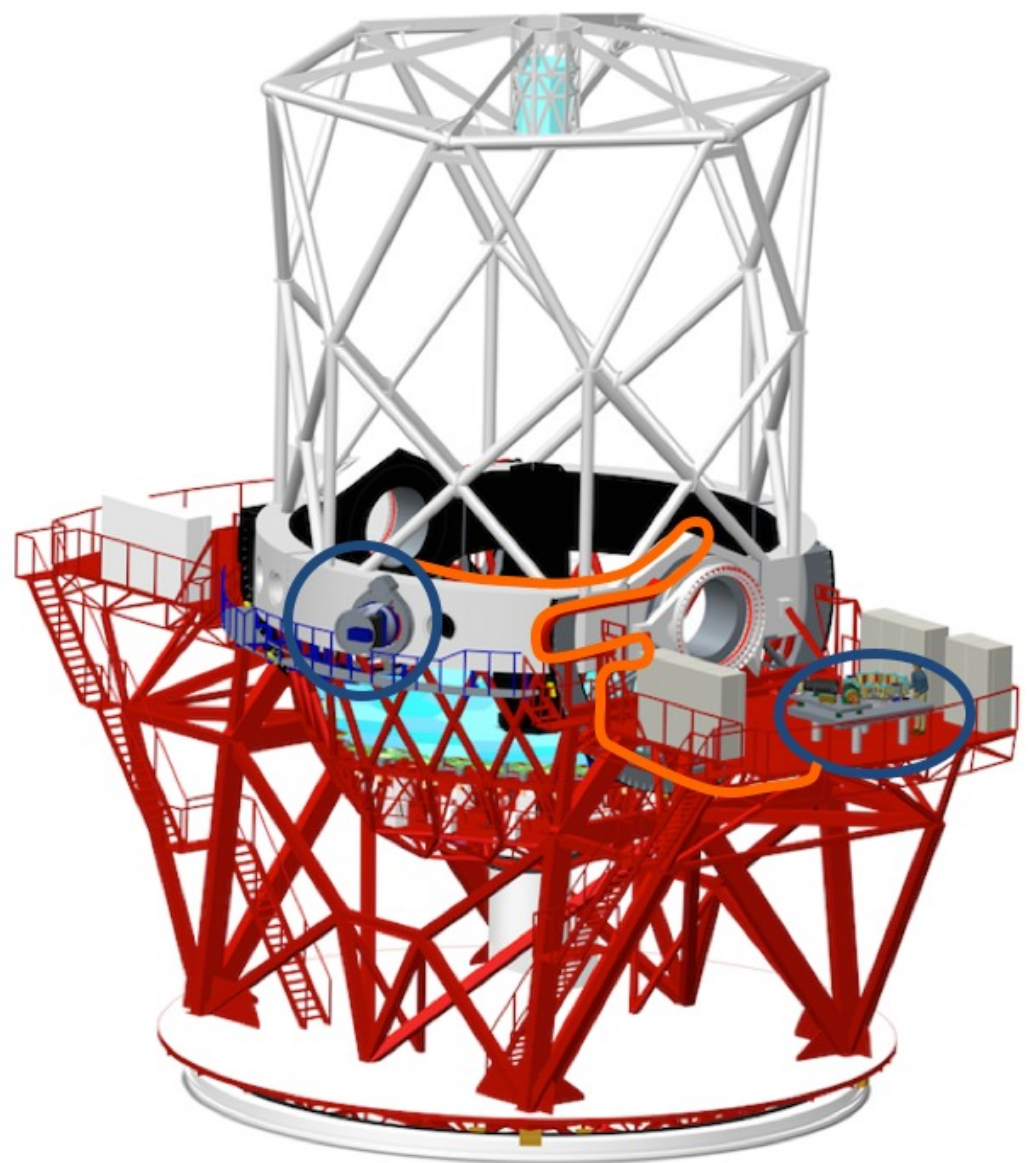

Figure 1: 3D view of the GTC telescope with the MEGARA Fiber-MOS unit placed at its Folded-Cassegrain F focus and the spectrograph sitting on the Nasmyth-A platform (both units encircled in blue). The approximate path of the $44.5 \mathrm{~m}-10 n g$ fiber link is outlined in orange. 


\subsection{Science drivers}

As a facility instrument, the design of MEGARA was aimed to be able to accommodate a broad range of scientific interests. This naturally led to the formation of a large and diverse Science Team, which is currently composed by 65 researchers mainly from the GTC consortium institutions (Spain, INAOE/UNAM in Mexico, University of Florida) but also from elsewhere. Broadly speaking, the scientific interests of the MEGARA Science Team, which ultimately define the characteristics of the instrument as outlined above, can be grouped in two categories, (1) the study of Galactic and extragalactic extended nebulae and (2) the study of multiple compact sources with intermediate-to-high number densities in the sky. The light grasp of GTC combined with MEGARA ensures limiting magnitudes faint enough to yield such number densities on the sky for many of our scientific topics of interest.

Category (1) includes the study of Galactic Planetary Nebulae, nearby galaxies, and the high-redshift IGM while in category (2) we find Galactic open stellar clusters, resolved stellar populations in Local Group galaxies, intermediateredshift dwarf and starburst galaxies, and high-redshift cluster galaxies (see Figure 2). More details on the science drivers of the MEGARA instrument have been published elsewhere ${ }^{1,2,3,4,5}$. Some of these scientific goals are now pursued using either Open Time (2018B observations started on July $1^{\text {st }} 2018$ ) or Guaranteed Time observations. In Section 1.4 below we focus on the plans for the scientific exploitation of MEGARA as part of the Guaranteed Time available to the MEGARA Consortium institutions. More details on these planned observations and on the early scientific results obtained as part of the night-time commissioning observations acquired from June to August 2017 are given in an accompanying paper ${ }^{6}$.

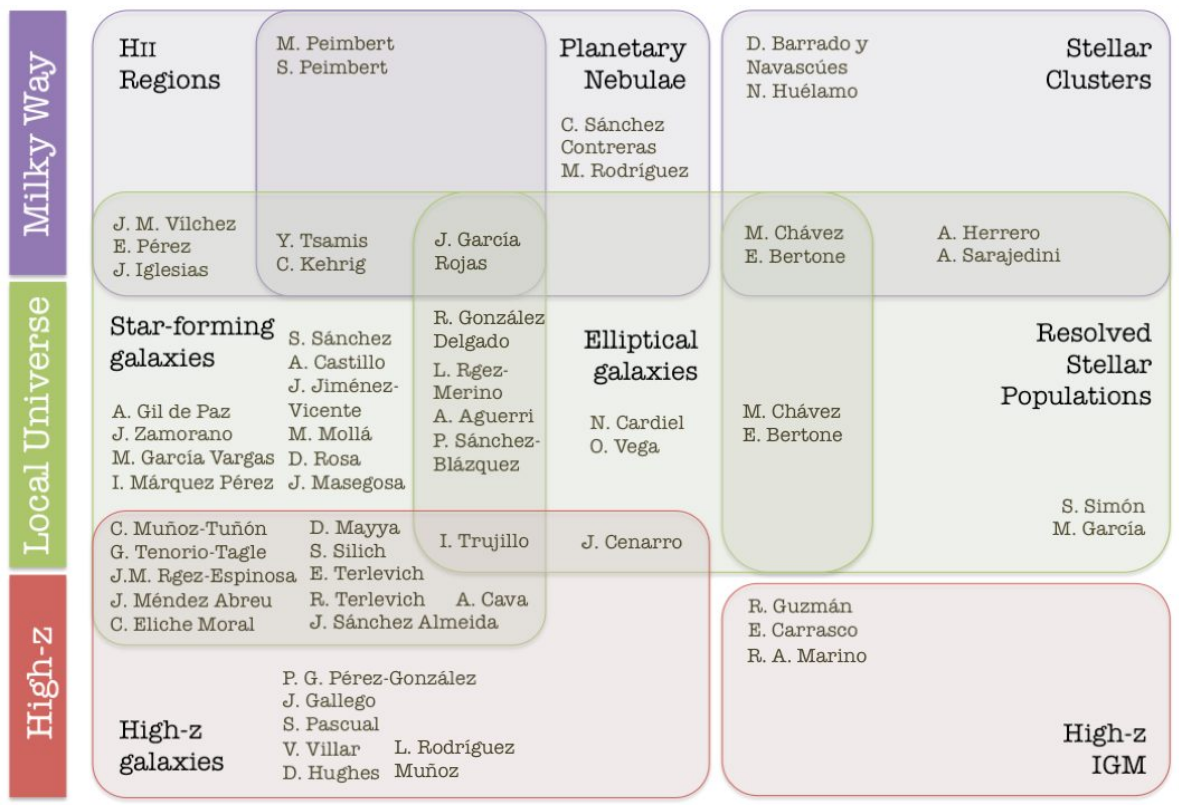

Figure 2: Main scientific interests of MEGARA Science Team members. These are also the main drivers that are behind the unique capabilities of the MEGARA instrument in terms of efficiency, resolution and versatility that have been put to test as part of the commissioning observations described in Section 5.

\subsection{Guaranteed Time scientific exploitation}

Among the drivers that led to the top-level requirements of the MEGARA instrument, the researchers of institutions of the MEGARA Consortium defined a series of scientific objectives to be pursued with the Guaranteed Time awarded by GRANTECAN to the UCM in payment for the in-kind contribution of the Consortium institutions to the Design, Construction and AIV of MEGARA. Below we provide a summary of each of these objectives. A detailed description of the targets to be observed as part of these scientific objectives for Guaranteed Time exploitation is given in the document of Reserved Targets that can be found at http://guaix.fis.ucm.es/megara/files/Reserved Targets Sent MEGARA.pdf. 
- MEGARA Galaxy Disks Evolution Survey (MEGADES): The goal of MEGADES is to study the stellar populations, chemical abundances and velocity ellipsoids of a sample of nearby galaxies (up to $40 \mathrm{Mpc}$ in distance) from the $\mathrm{S} 4 \mathrm{G}$ sample ${ }^{7}$ and it is the most ambitious project of those proposed for being observed as part of the MEGARA Guaranteed Time. The MEGADES-S4G sample includes a total of 215 galaxies (29 elliptical galaxies) with 150 of them having SDSS-DR7 optical data publicly available. Roughly $2 / 3$ of them show inclinations larger than $40^{\circ}$ (but lower than $70^{\circ}$ ) and can therefore be used to determine their velocity ellipsoids. In order to evaluate the limitations inherent to the study of the unresolved stellar populations of MEGADES-S4G galaxies with the LCB we will also carry out this study using resolved stellar populations in the disk of Local Group galaxy M33, for which both visibility and disk inclination are perfectly suited for carrying out this study using the MEGARA MOS at GTC. Finally, the results of this survey regarding the balance between in-situ and ex-situ processes in shaping the (effective) star formation, chemical and dynamical history of M33 and the S4G survey galaxies will be compared with the results of the ESA Gaia mission for our own Milky Way.

- Spectroscopic Study of Compact Stellar Clusters (CSCs) and their surroundings in nearby galaxies: We propose to observe spectroscopically a sample of galaxies containing a rich population of CSCs, with the primary goal of age-dating these clusters. The ages would be obtained by comparing the observed spectra containing characteristic absorption and/or emission lines with the spectra from population synthesis models ${ }^{8}$. The derived age distribution of clusters would help to identify major past events of star formation ${ }^{9}$. As the interaction between galaxies is the principal driver of violent star formation, age distribution of CSCs could be used to determine the mass assembly history of the sample galaxies. This will be done on three sets of targets: a sample of very nearby galaxies where the sample of CSCs will be covered using the MOS, nearby galaxies with extended starburst activity to be observed in the LCB mode, and a sample of Wolf-Rayet bubbles in M81, also to be done with the LCB.

- The Chemical Composition of Photoionized Nebulae: The spectra of H II regions and planetary nebulae (PNe) can be analyzed with very similar techniques to derive the abundances of several elements. The comparison between these abundances have led to one of the most important problems in the field that we will tackle with MEGARA@GTC, that is the discrepancy between the abundances implied by collisionally-excited lines and the weak recombination lines that can be observed for some ions ${ }^{10,11}$, notably $\mathrm{O}^{++}$. All $\mathrm{H}$ II regions and PNe where O II recombination lines have been measured show this discrepancy, with the abundances derived with recombination lines being larger than those obtained with collisionally-excited lines with abundance discrepancy factors ranging between ${ }^{12} \mathrm{ADFs} \sim 2-100$. Some of the Galactic targets to be observed as part of this project include NGC2022, Vy1-2, NGC6572, Hu2-1, NGC6778, NGC6884, NGC6891, NGC7026, NGC7635 along with extragalactic HII regions NGC2363, NGC5461, NGC5471 and specific areas within the Local Group galaxies M33 and IC10.

- Chromospheric activity and age of Solar analogs in Open Clusters: The ultimate goal here is to provide a reliable age determination method for field Solar analogs to be used in the search for potential extrasolar SunEarth systems. Our first aim is to use the chromospheric activity as an age indicator, so we will start from a calibrating sample of solar-type stars in Open Clusters with known ages. We will measure the chromospheric emission of the $\mathrm{Ca} \mathrm{H}$ and $\mathrm{K}$ lines at $3969 \AA$ and $3933 \AA$ in these solar-type stars using a selected sample of 20 open clusters of roughly Solar metallicity and ages in the range from $10 \mathrm{Myr}$ and $4 \mathrm{Gyr}^{13}$.

- Dissecting z 2-3 He II emitters: Spectral templates for the sources of the Cosmic Dawn: We will use the MEGARA IFU to obtain spatially and spectrally resolved information on the $\mathrm{He}$ II emission for spectroscopically confirmed He II emitters selected from cosmological surveys (e.g. VUDS). From these data, we will derive the total He II-ionizing photon budget (free from aperture effects) and constrain the ionization mechanism for the He II line. A spaxel-by-spaxel analysis of the He II line, and additional emission lines (e.g.,

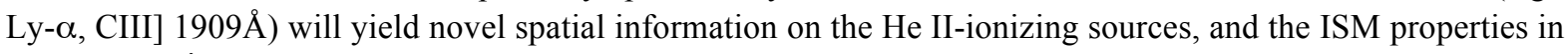
our He II-emitters.

- Chemodynamics of metal-poor extreme Emission Line Galaxies at intermediate z: We will obtain 2D spectroscopy of the ionized gas in three Extremely Metal-Poor (XMP) that are also extreme Emission Line Galaxies (EELGs) located at intermediate redshift $(\mathrm{z}=0.35-0.7)$. These objects have been selected from the zCOSMOS-20k survey ${ }^{14}$ because of their intense and high-EW [O III] emission lines and very low metallicities as obtained from their [O III] electron temperatures. Our aim is assessing whether these objects are metal-poor 
objects in formation resembling the galaxy building blocks in the early Universe or evolved interacting systems in which starburst activity, disrupted morphology, and the dilution of the gas-phase metallicity are owing to the in-fall of chemically unpolluted gas.

- Constraining Wolf-Rayet stars In Extremely Metal-Poor Galaxies: We will take advantage of the catalogue of Wolf-Rayet galaxies in SDSS having a well understood selection function to observe with the LCB of MEGARA a subsample of extremely metal-poor objects $\left(12+\log (\mathrm{O} / \mathrm{H})<\mathrm{Z}_{\odot} / 10\right)$. The observations of these galaxies using integrated spectra has brought to light large differences between model predictions and observations with respect to the strength of the Wolf-Rayet features at low metallicity. These observations will provide enough spatial and spectral information to characterize the Wolf-Rayet population and the ionic abundances and give new and precise constraints to the models in this regime.

- Exploring the physical properties of shocked ionized gas in interacting galaxies: Interactions of galaxies play an important role in the evolution of galaxies. In particular, a high-velocity encounter can lead to shocks where the interstellar material is heated to very high temperatures and presents properties where multiple kinematical components are apparent. Stephan's Quintet, a paradigm of this kind of phenomena showing a bar-like shocked

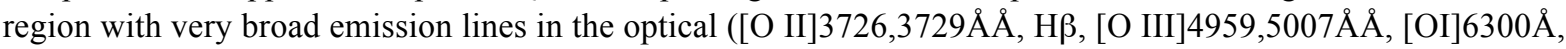

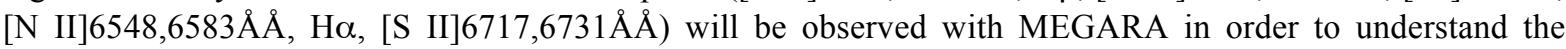
physical mechanisms that govern the physical properties of the ionized interstellar gas under these conditions.

- Galactic Winds in nearby galaxies: We will characterize neutral and ionized winds in nearby galaxies previously identified in the CALIFA survey as showing strong $\mathrm{NaD}$ absorption. We will also add to these observations the study of a sample of (more active in terms of massive star formation) northern ULIRGs ${ }^{15}$.

- Dwarf galaxies in Cosmological fields: We will target dwarf emission-line galaxy candidates at intermediate-tohigh redshifts in Cosmological fields using the MEGARA MOS in order to determine their internal kinematics along with basic parameters such as their ionized-gas chemical abundances (from the [NII] $6584 \AA / \mathrm{H} \alpha$ line ratio) and SFR measurements. These observations will be one of the first attempts to address the evolution of the faint end of the luminosity function based on a large sample of spectroscopically-confirmed targets. Again, the faint limiting narrow-line fluxes to be achieved in this program ensures a high-density of objects and an optimal use of the MOS FoV.

- Gravitational Lenses: We will characterize a sample of QSOs affected by gravitational macro/micro-lensing using the MEGARA LCB, which allows to simultaneously observe most of the QSOs components. The spectral resolution of MEGARA in conjunction with the large aperture of GTC offers a unique opportunity to observe in detail the presence of microlensing both in the continuum and in the BLR spectral lines of these QSOs.
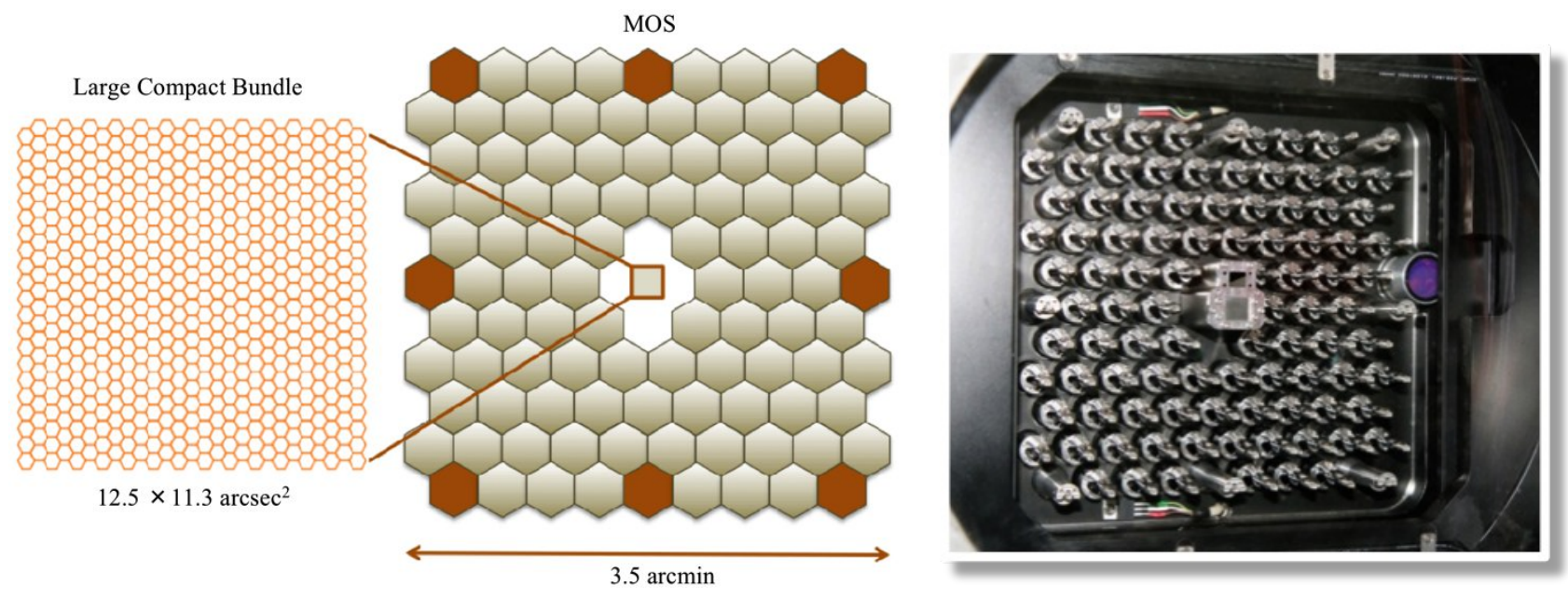

Figure 3: LCB and MOS in the focal plane of MEGARA at the Folded-Cass F focus of GTC. Left: Layout of the monolithic microlens array of the LCB placed at the optical axis of the instrument. Center: Hexagons representing the patrol areas of the 92 robotic positioners of the MEGARA MOS (in light grey) along with the positions of the eight sky bundles that are mounted along the LCB pseudo-slit (in orange). Note that the actual patrol areas are overlapping circular regions of 28 arcsec in diameter, while the distance between adjacent positioners is 24.5 arcsec. Right: Photograph of the MEGARA focal plane before the field lens was installed at the UCM-LICA laboratory. 


\section{FIBER-MOS UNIT}

In this section we describe in more detail the characteristics of the Fiber-MOS unit of MEGARA. This unit constitutes the part of the instrument that is attached to the Folded-Cass F focus of GTC and includes the following subsystems: field lens, LCB, MOS, boresight telescope, focal-plane cover, and adapter to the GTC Folded-Cassegrain F rotator.

\subsection{Field lens}

In order to provide a telecentric configuration for both LCB and MOS modes, but mainly for the MOS, early on in the design we included a Field Lens that now allows all robotic positioners, independently of the distance to the instrument optical axis, to be mounted in a common, flat mechanical frame that would be also confocal with the LCB. This lens introduces basically no power neither changes the plate scale at the instrument focal plane.

\subsection{Focal Plane cover}

Right after the field lens, the instrument includes a cover that allows to block each of the two halves of the instrument focal plane independently (split along the vertical direction of the field as shown in Figure 3). The cover was designed to block the light as close to the surface of LCB and MOS robotic positioners microlens-arrays as possible. In this way, while the central three columns of spaxels (63 spaxels, i.e. $\sim 10 \%$ of the FoV) are partly exposed to light, about 45 per cent of the fibers (252 spaxels) remain completely exposed and 45 per cent (the remaining 252 spaxels) are fully blocked by each of the two sides of the focal plane cover. Each blocked fiber leads to a gap of 12 pixels in between the centers of each two consecutive illuminated fibers on the detector. This reduces the cross-talk between adjacent fibers to null. See Figure 4 for a graphical description of the effect of the observations with and without the focal plane cover in the case of the LCB (left panel) and the MOS (right panel).
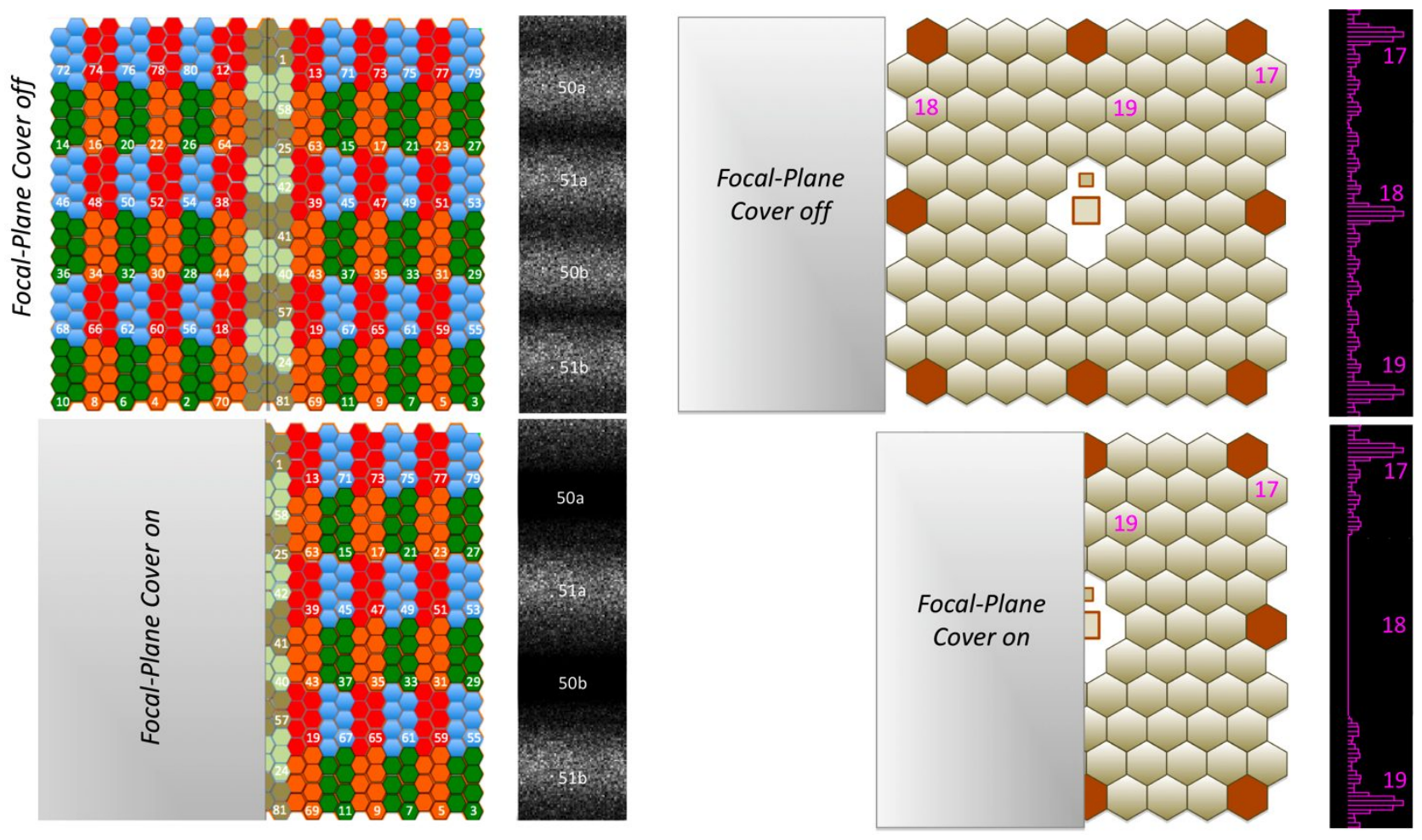

Figure 4: Left: Layout of the different spaxels in the LCB focal plane that are exposed and blocked to light when the focal-plane cover is used (only the left-cover configuration is shown in this example). Note that spaxels corresponding to minibundles (from top to

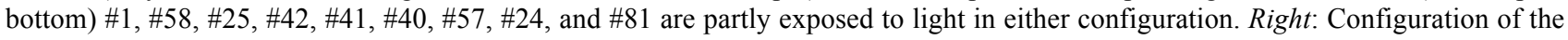
left cover when used in its MOS mode. The position in the FoV and corresponding simulated light profiles of the MOS pseudo-slit spectra on the detector for robotic-positioner MOS minibundles \#17, \#18 and \#19 are shown in the leftmost panels. 


\subsection{Large Compact Bundle}

The LCB IFU of MEGARA is composed by the monolithic LCB microlens array manufactured by A $\mu$ S, the mechanical frame and the fibers that are attached to the LCB microlens array and to eight sky mini-bundles. The LCB microlens array changes the focal ratio from the input $\mathrm{F} / 17$ to $\mathrm{F} / 3$, for minimizing Focal-Ratio Degradation (FRD hereafter) throughout and at the exit of fiber link for our F/3 spectrograph collimator. This lens array is composed of 567 microlenses arranged in the focal-plane as shown in Figure 3. Details of different parts of the LCB, including the mechanical frame and the lens array and the gluing station now installed at the UCM LICA laboratory (used to assemble all mechanical frame, all 567 IFU fibers, and the lens array) are shown in Figure 5. Each of the 567 fibers of the LCB and the 56 sky fibers are all $100 \mu \mathrm{m}$ in core size, $140 \mu \mathrm{m}$ with the cladding and $170 \mu \mathrm{m}$ with the external coating.
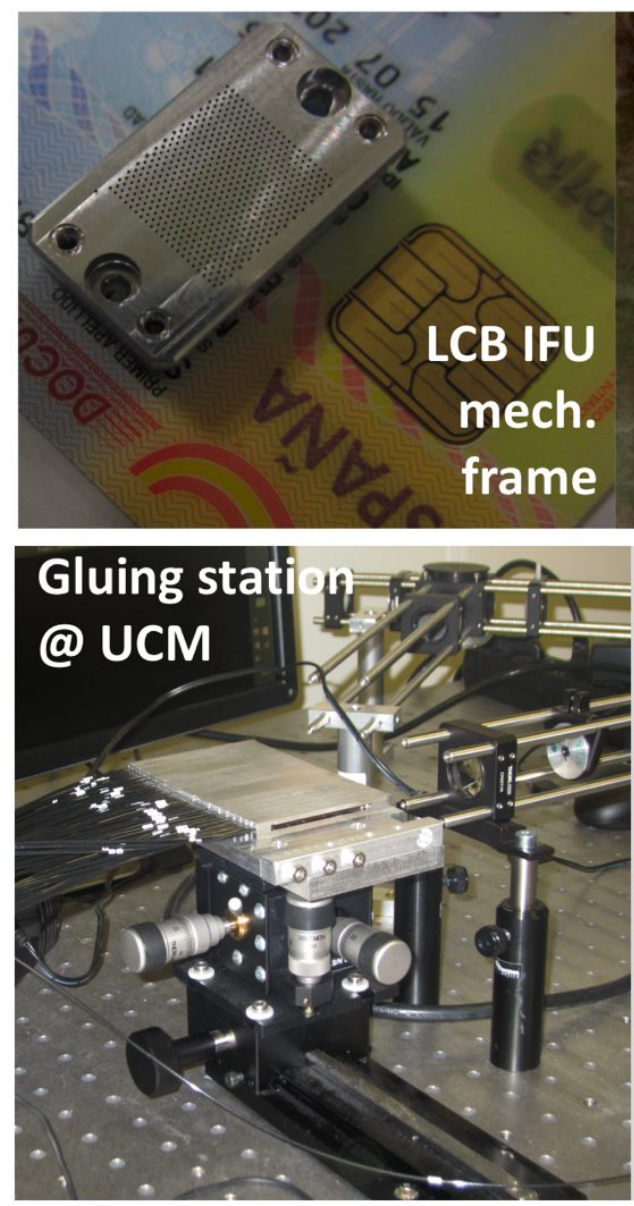
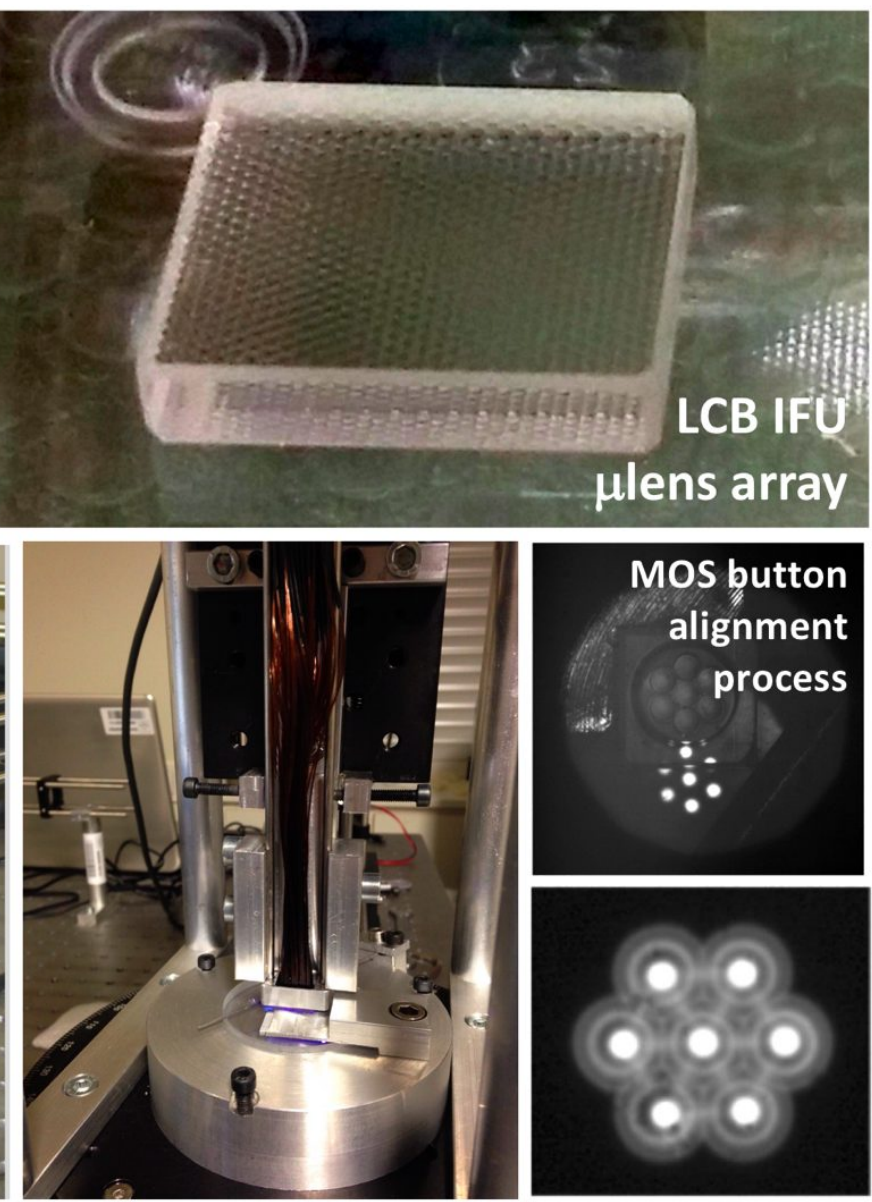

Figure 5: Top: Large Compact Bundle IFU mechanical frame (left) and corresponding 567-spaxel lens array from A $\mu$ S (right). Bottom: Gluing station developed for the Laboratorio de Instrumentación Cientifica Avanzada (LICA) at UCM that was used for gluing the LCB lens array to its 567-fiber bundle (left and center) and each of the 92 individual 7-spaxel microlens arrays of the MOS to its corresponding 7-fiber minibundle (right).

\subsection{MOS}

Around the LCB and extending to a field of $3.5 \times 3.5 \operatorname{arcmin}^{2}$, still within the flat and unvignetted focal plane of GTC at its FC-F focus, a total of 92 robotic positioners are arranged in a hexagonally-packed rectangular pattern (see Figure 3). Each of the positioners holds a 7-fiber minibundle with identical $100 \mu \mathrm{m}$-core fibers to those used in the MEGARA LCB. In order to place each of these 92 positioners in any specific point within the $23.1 \mathrm{~mm}(28 \operatorname{arcsec})$ diameter of the corresponding patrol area, a combination of two rotations is used, namely R1, for which the positioner rotates around its 
symmetry axis, and R2, where the arm that holds the button with the 7-fiber minibundle rotates around an axis that is offset $5.8 \mathrm{~mm}$ from the symmetry axis. This interpolation of this combined motion and the range of operation of the two rotations $\left(0-365^{\circ}\right.$ for $\mathrm{R} 1$ and $0-185^{\circ}$ for $\left.\mathrm{R} 2\right)$ allows fully covering the circular patrol area of 28 arcsec in diameter mentioned above. The detailed system engineering needed to ensure the fulfillment of the tilt requirements for both rotation axes along with the commissioning results regarding the alignment with the telescope pupil and XY positioning of the fiber minibundles across the entire MOS FoV are described in an accompanying paper by Pérez-Calpena et al. $(2018)^{16}$.

\subsection{Boresight telescope}

In addition to the aforementioned subsystems, the Fiber-MOS unit also includes a boresight telescope (the right side of Figure 3) that, attached to an off-the-shelf CCD camera, allowed us imaging the telescope pupil during the integration of MEGARA at GTC so to ensure a precise alignment of the instrument optical axis and the telescope optical axes ${ }^{16,17}$. The boresight telescope can be seen in Figure 3. As detailed by Pérez-Calpena et al. $(2018)^{16,17}$, we identified a tilt in the $\mathrm{M} 2-\mathrm{M} 3$ axis of 5 arcmin. This result was communicated to GTC personnel who confirmed our measurements.

\subsection{Fiber link}

Although not properly part of the Fiber-MOS unit, the fiber link can be considered as an extension of the LCB and MOS subsystems that connect the Fiber-MOS to the MEGARA spectrograph placed at the Nasmyth A platform of GTC. In the left panel of Figure 6 we show the fibers coming out from the back of the focal plane of MEGARA. All fibers are arranged in minibundles of 7 fibers, each protected by its own $1.2 \mathrm{~mm}$ flexible tube. These are clearly visible in Figure 6 both for the $92+8$ spare bundles of the MOS and sky bundles and the 81 packed bundles in the center corresponding to the LCB. The total length of the fiber link from the FC-F focal plane to the spectrograph entrance focal plane is $44.5 \mathrm{~m}$, which leads to more than $56 \mathrm{~km}$ of optical fiber. The path followed by the fibers (see also Figure 1) includes the FC-F rotator, the telescope ring, the elevation rotator, and the Nasmyth A platform itself.
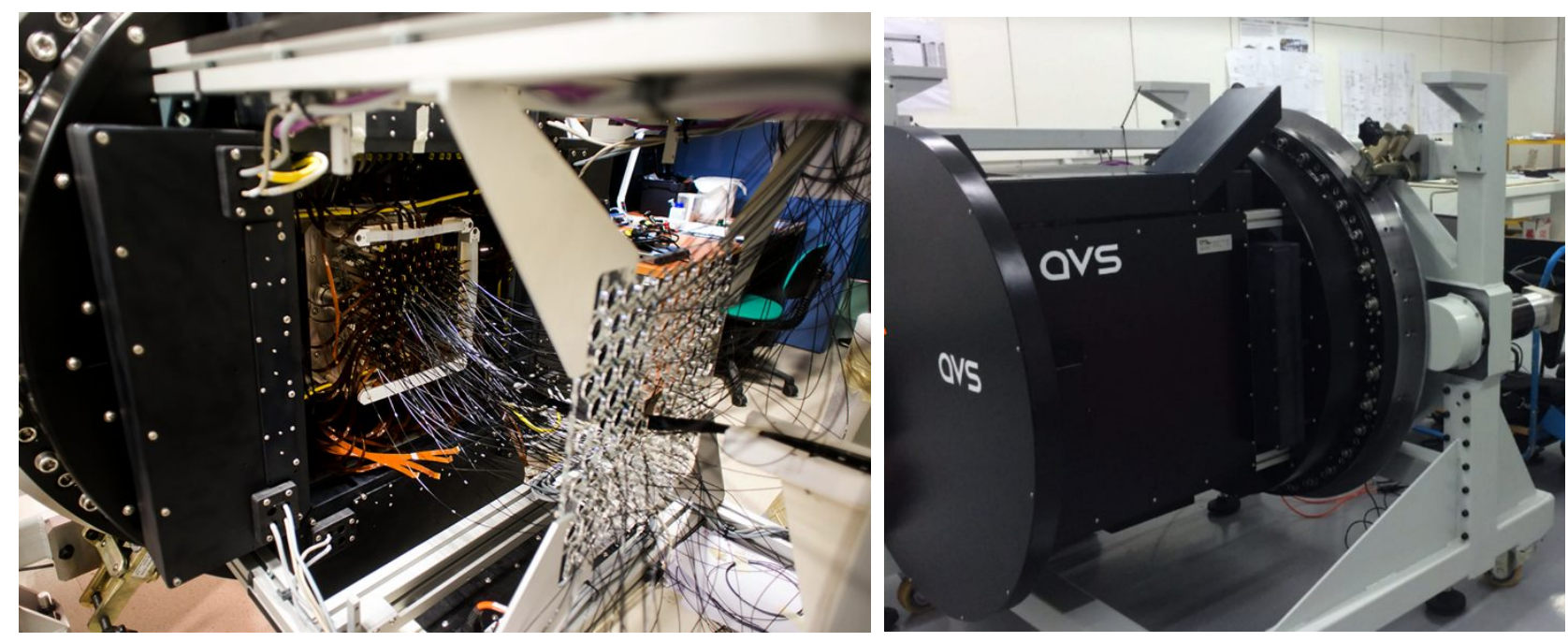

Figure 6: Left: Inside view of the Folded-Cassegrain Rotator Adapter where all FC-F elements of MEGARA are held together and aligned. This adapter also provides the elements for a proper fiber routing from the focal plane towards the FC-F rotator. The routing of both the $92+8$ MOS and sky bundles and the pack of 81 LCB bundles are clearly seen in this photo taken at LICA. Right: Photo of the Folded-Cassegrain Rotator Adapter taken at the GTC clean room, right before its installation at the FC-F focus of the telescope. 


\subsection{Folded-Cassegrain rotator adapter}

All previous subsystems are held together and attached to the Folded-Cass F rotator by a structure called FoldedCassegrain rotator adapter, which is composed by the optical assembly and the auxiliary assembly.

The optical assembly comprises the field lens, the focal plate and the focal plane cover envelope, with the corresponding enclosure and alignment structural components.

The auxiliary assembly is supported independently of the optical assembly by 6 tubular legs welded to an intermediate flange, which is screwed to the interface flange without affecting the optical assembly positioning and aligning.

The total weight of the elements that are attached to the FC-F rotator, including the fibers and feeding cables, is $420 \mathrm{~kg}$. This also includes the electronic boxes that contain the positioner controllers and that are hanged off the adapter as well as an additional box that contains the positioners' power supplies (see Figure 6).

\section{SPECTROGRAPH}

The MEGARA spectrograph is located at the Nasmyth A platform of GTC on an optical bench that rests in a total of 5 pneumatic legs. In addition to the fiber link described above, also the GTC glycol-water circuit and power and control connections arrive to this optical bench (Figure 7). The different hardware subsystems of the MEGARA spectrograph are the pseudo-slits and associated interchange and focusing mechanism, the collimator, camera, pupil elements (VPH), the VPH wheel and the MEGARA cryostat and detector. In this section we describe in more detail each of these subsystems.

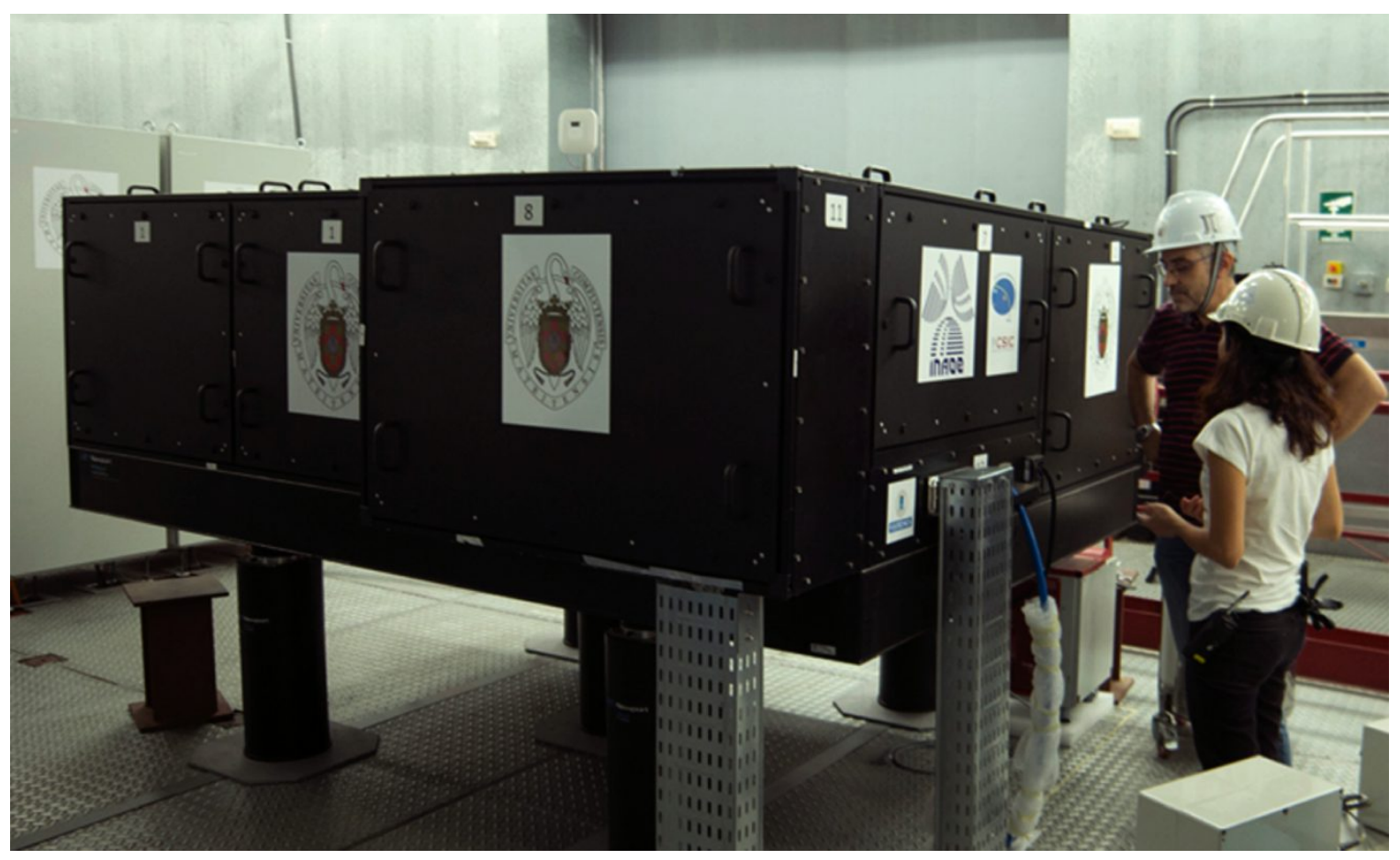

Figure 7: Photograph of the MEGARA optical bench at the Nasmyth A platform of GTC on top of which the MEGARA spectrograph is located. The fiber link arrives from under the Nasmyth A platform (metal protections are seen at the bottom center portion of the photograph) along with the power and control connections (metal protection at right) and the glycol water (blue tube at bottom right). 


\subsection{Pseudo-slits}

At the end of the fiber link the 623 fibers from the LCB (sky bundles included) and the 644 fibers from the 92 MOS minibundles arrive to the MEGARA spectrograph arranged in two different pseudo-slits. These two pseudo-slits are placed at the entrance focal-plane of the spectrograph (located in front of the first lens of the MEGARA collimator) and can be interchanged by means of a linear stage that moves them perpendicular to the position of the fibers, see Figure 8 . This mechanism also acts as focusing mechanisms by moving the pseudo-slits along the direction of the fibers to compensate for the different foci as a function of the VPH element being used and the spectrograph temperature (mainly) but also of the pseudo-slit in use.

The two pseudo-slits are telecentric and follow a curved focal plane with a ROC of $1075 \mathrm{~mm}$ and a size (tangential to the curve) of $119 \mathrm{~mm}$. In order to follow this curvature, the fibers of the LCB (MOS) pseudo-slit are distributed in 17 (19) flat boxes on which all the fibers in each box were polished together.
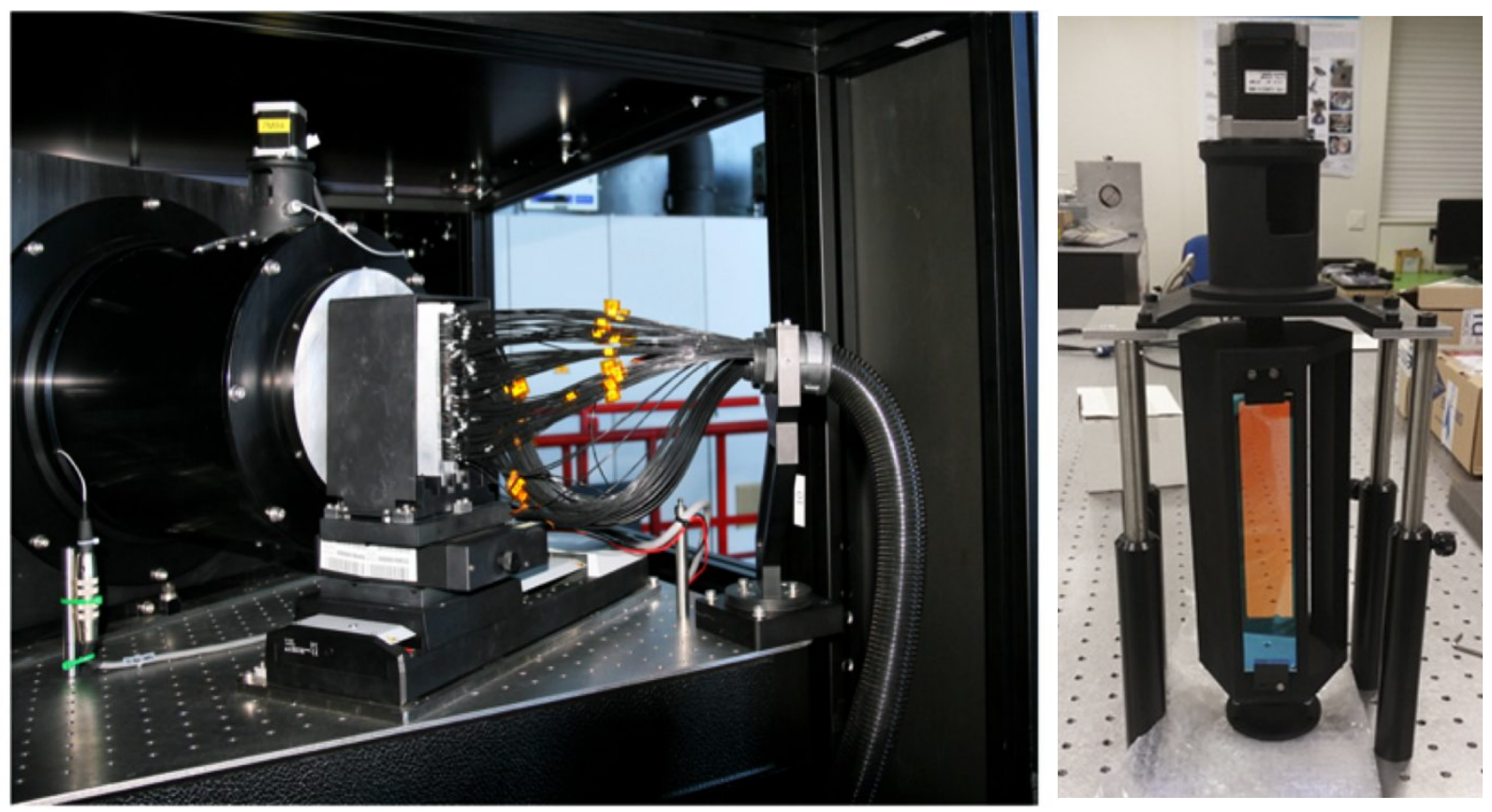

Figure 8: Left: Inside view of the MEGARA optical bench with the two fiber bundles arriving to the entrance focal-plane of the spectrograph. The collimator is also visible in this image with the upper part of the rotating shutter visible in on top of the collimator. Right: View of the rotating shutter at LICA before its integration with the MEGARA collimator.

\subsection{Collimator}

The MEGARA Spectrograph is a fully refractive optical system. The collimator is composed by 5 lenses ( 1 singlet and two doublets) with the first lens of the collimator being the only aspherical surface of the instrument, which is one of the smallest lenses in the system (140mm diameter and made of PBM2Y glass). This is an F/3 collimator with a focal length of $484.4 \mathrm{~mm}$ (see left panel of Figure 8). A rotating slit shutter is placed right beyond the first collimator lens. The shutter has three positions: the open closed position and the so-called red and blue positions. These two "open" positions correspond to a long-pass, order-sorting (OS) filter, and a short-pass filter, respectively. The former is needed for removing the contamination from second order when observing at long wavelengths, while the latter was included in the detailed design as early tests done at the lab showed that the HD technology used for the medium-resolution (MR) VPHs (where the final lines/mm on the VPH gelatin are recorded in two sets with half the lines $/ \mathrm{mm}$ ) led to some level of contamination from light with wavelengths slightly over half those being observed. An image of the shutter before being installed in the instrument is shown in the right panel of Figure 8. 
The parallel beam coming out from the collimator then defines the position of the pupil where the disperser elements (VPHs in our case) are placed. The different VPHs used and how they are placed in the pupil is described in Section 3.4 below.

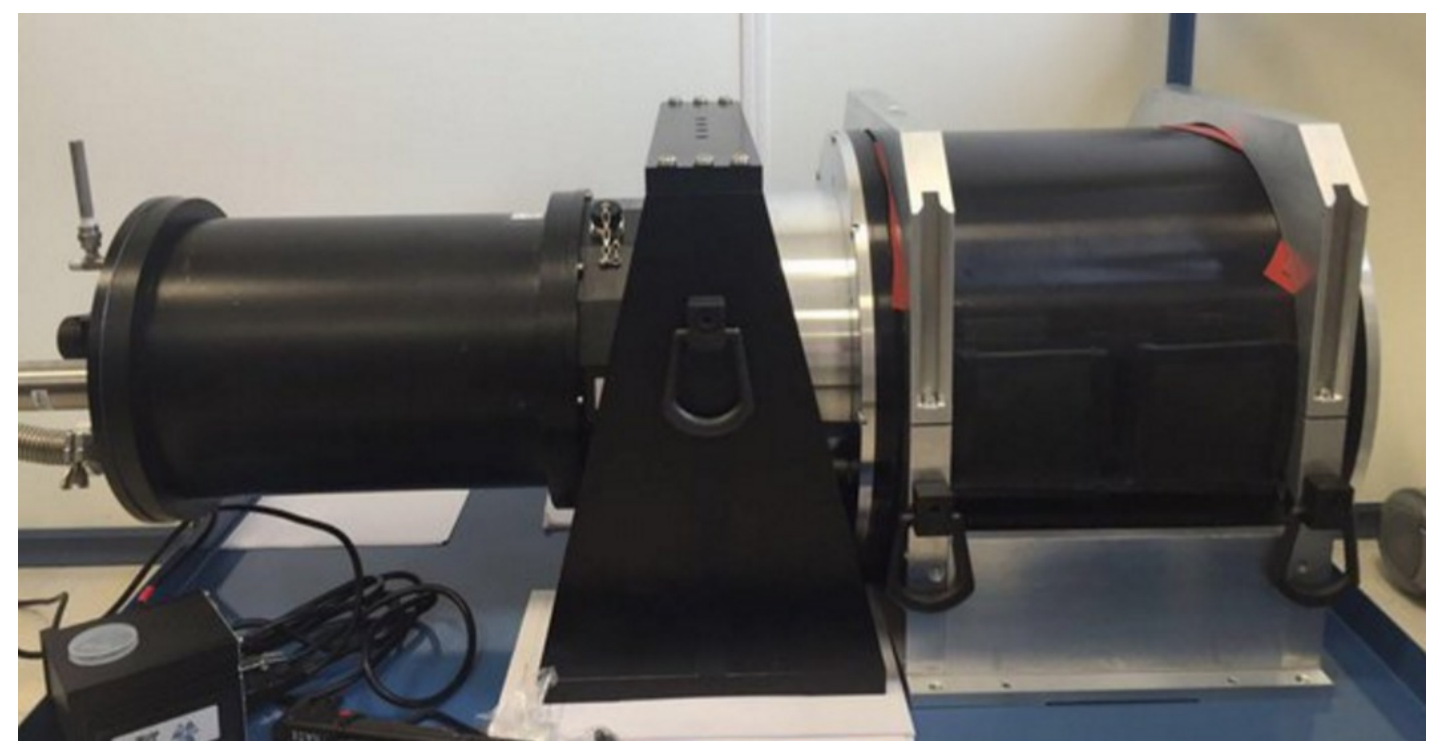

Figure 9: Camera-cryostat assembly seen at the LICA before being integrated with the rest of the MEGARA spectrograph. The camera is at the right and the cryostat is at the left. The last lens of the camera also acts as window for the LN2 open-cycle cryostat, where the E2V 231-84 model CCD is located. The entire camera-cryostat assembly has been manufactured at INAOE in Puebla (Mexico).

\subsection{Camera, cryostat and detector}

Once the beam passes through the VPH it goes to the camera that focuses the light onto the detector. The angle between collimator and camera is fixed and equal to $68^{\circ}$. This is equivalent to $34^{\circ}$ between the collimated beam and the normal to the VPH grating. The camera (seen already integrated in Figure 9) is composed by two doublets and 3 singlets, being the last lens also the cryostat window. Each of the two doublets includes a $\mathrm{CaF}_{2}$ lens, the other materials being BSM51Y and BAL15Y glasses, while the singlets are made of $\mathrm{CaF}_{2}, \mathrm{~S}-\mathrm{LAH} 55 \mathrm{~V}$ and S-NBH8. The camera has a focal ratio of F/1.5 and its focal length is $245.9 \mathrm{~mm}$.

The image field is $61.4 \mathrm{~mm} \times 61.4 \mathrm{~mm}$ to cover the $4 \mathrm{k} \times 4 \mathrm{k} 15 \mu \mathrm{m}$ pixels of the MEGARA CCD detector. This field allows to focus the spectra generated by all fibers in the pseudo-slits (the MOS pseudo-slit is slightly larger than the LCB one) for output angles from the pupil in the range $\pm 7^{\circ}$, for which the optical design safely meets the image quality requirements. As shown in Section 4, the performance obtained in terms of image quality and resulting spectral resolution during integration and commissioning confirmed that the instrument meets both these requirements. The detector used in MEGARA to cover this field is a E2V 231-84-1-E74 (grade 1) deep-depleted CCD model with "astro multi-2" coating. This detector and the corresponding readout electronics yield a readout noise $<3 \mathrm{e}^{-}$and a dark current $<2 \mathrm{e}^{-}$/hour.

The MEGARA CCD is hosted in a LN2 open-cycle cryostat designed and built at INAOE. This is a 7-liter cryostat that weights $23 \mathrm{~kg}$ and works at an operating temperature of $158 \mathrm{~K}$ and a hold time of 40 hours (see Figure 7). It has been in operating conditions of pressure and temperature continuously since its integration at GTC back in April 2017 (see Section 4.1 for a detailed description of the instrument integration at GTC). 


\subsection{VPHs and VPH wheel}

In order to disperse the light, the MEGARA spectrograph makes use of a series of $18 \mathrm{VPH}$ gratings that yield spectral resolutions between $\mathrm{R}=6,000-20,000$ across the entire optical range from $365-970 \mathrm{~nm}$. These VPHs must be located at the pupil and must be able to accept a beam of $160 \mathrm{~mm}$ in diameter, which is the size of the spectrograph pupil.

The requirement in spectral resolution for the different types of VPHs in terms of $\mathrm{R}_{\mathrm{EED} 80}\left(\lambda / \Delta \lambda_{\mathrm{EED} 80}\right)$ were 5,500, 10,000 and 17,000 (respectively for so-called LR, MR and HR VPHs) for both the LCB and MOS observations. When expressed in terms of the FWHM of unresolved lines in the extracted (1D) spectra the requirements for the spectral resolution become $\mathrm{R}_{\mathrm{FWHM}} \sim 6,000$ (low resolution; LR), $\mathrm{R}_{\mathrm{FWHM}} \sim 12,000$ (mid-resolution; MR) and $\mathrm{R}_{\mathrm{FWHM}} \sim 18,700$ (high resolution; HR). The commissioning results show that both all the LR and MR requirements are fulfilled and even exceeded in the case of the HR VPHs, which effectively reached $\mathrm{R}_{\mathrm{FWHM}} \sim 20,000$. With regard to the wavelength coverage MEGARA offers full optical coverage with both LR and MR VPHs. The ranges covered by the HR VPHs are 640-680 $\mathrm{nm}$ for the HR-R (which is centered around the $\mathrm{H \alpha}$ line at $656.3 \mathrm{~nm}$ ) and 838-863 $\mathrm{nm}$ for the HR-I VPH (centered at the CaT lines).

Table 2. Results of the instrument commissioning regarding the spectral performance of the different MEGARA VPHs. $\lambda \min _{1}\left(\lambda \max _{2}\right)$ represents the minimum (maximum) wavelength covered for at least one of the LCB fibers, while $\lambda \mathrm{c}$ represents the central wavelength and $\lambda \min (\lambda \max )$ the minimum (maximum) wavelength common to all LCB fibers.

\begin{tabular}{|c|c|c|c|c|c|c|}
\hline VPH & $\lambda \min _{1}(\AA)$ & $\lambda \min (\AA)$ & $\lambda c(\AA)$ & $\lambda \max (\AA)$ & $\lambda \max _{2}(\AA)$ & Disp. $(\AA /$ pix $)$ \\
\hline LR-U & 3640,04 & 3654,32 & 4025,90 & 4391,88 & 4417,33 & 0,195 \\
\hline LR-B & 4278,43 & 4332,05 & 4785,32 & 5199,96 & 5232,02 & 0,230 \\
\hline LR-V & 5101,13 & 5143,74 & 5678,63 & 6168,19 & 6206,04 & 0,271 \\
\hline LR-R & 6047,62 & 6096,54 & 6729,61 & 7303,21 & 7379,88 & 0,321 \\
\hline LR-I & 7166,47 & 7224,11 & 7976,31 & 8640,37 & 8822,29 & 0,380 \\
\hline LR-Z & 7978,45 & 8042,74 & 8873,16 & 9634,92 & 9692,58 & 0,421 \\
\hline MR-U & 3911,99 & 3919,81 & 4102,87 & 4282,17 & 4289,11 & 0,092 \\
\hline MR-UB & 4217,44 & 4226,38 & 4429,44 & 4625,79 & 4633,65 & 0,103 \\
\hline MR-B & 4575,84 & 4585,66 & 4809,46 & 5025,07 & 5033,66 & 0,112 \\
\hline MR-G & 4952,15 & 4963,22 & 5208,79 & 5445,00 & 5454,62 & 0,126 \\
\hline MR-V & 5369,03 & 5413,11 & 5664,96 & 5923,90 & 5659,56 & 0,135 \\
\hline MR-VR & 5850,19 & 5894,23 & 6165,79 & 6448,52 & 6468,52 & 0,148 \\
\hline MR-R & 6228,15 & 6243,10 & 6560,33 & 6865,26 & 6878,27 & 0,163 \\
\hline MR-RI & 6748,88 & 6764,58 & 7109,81 & 7440,85 & 7454,46 & 0,172 \\
\hline MR-I & 7369,39 & 7386,53 & 7765,14 & 8127,95 & 8142,75 & 0,189 \\
\hline MR-Z & 8787,88 & 8810,52 & 9274,84 & 9698,97 & 9740,20 & 0,222 \\
\hline HR-R & 6397,62 & 6405,61 & 6602,59 & 6797,14 & 6804,87 & 0,098 \\
\hline HR-I & 8358,64 & 8380,20 & 8626,01 & 8882,38 & 8984,87 & 0,130 \\
\hline
\end{tabular}


See Table 2 above for a description of the spectral ranges for all VPHs as derived from the MEGARA commissioning observations. Note that this table includes both the range covered by at least one fiber and the spectral window common to all fibers, which is the nominal spectral coverage quoted in the project documentation and in the GTC webpages. In Figure 10 we show a photograph of some of the VPHs of MEGARA mounted on the VPH wheel (left) and the resolving power vs. wavelength coverage of all VPHs in the case of the LCB mode. The R vs. $\lambda$ curves in the case of the MOS are almost identical..
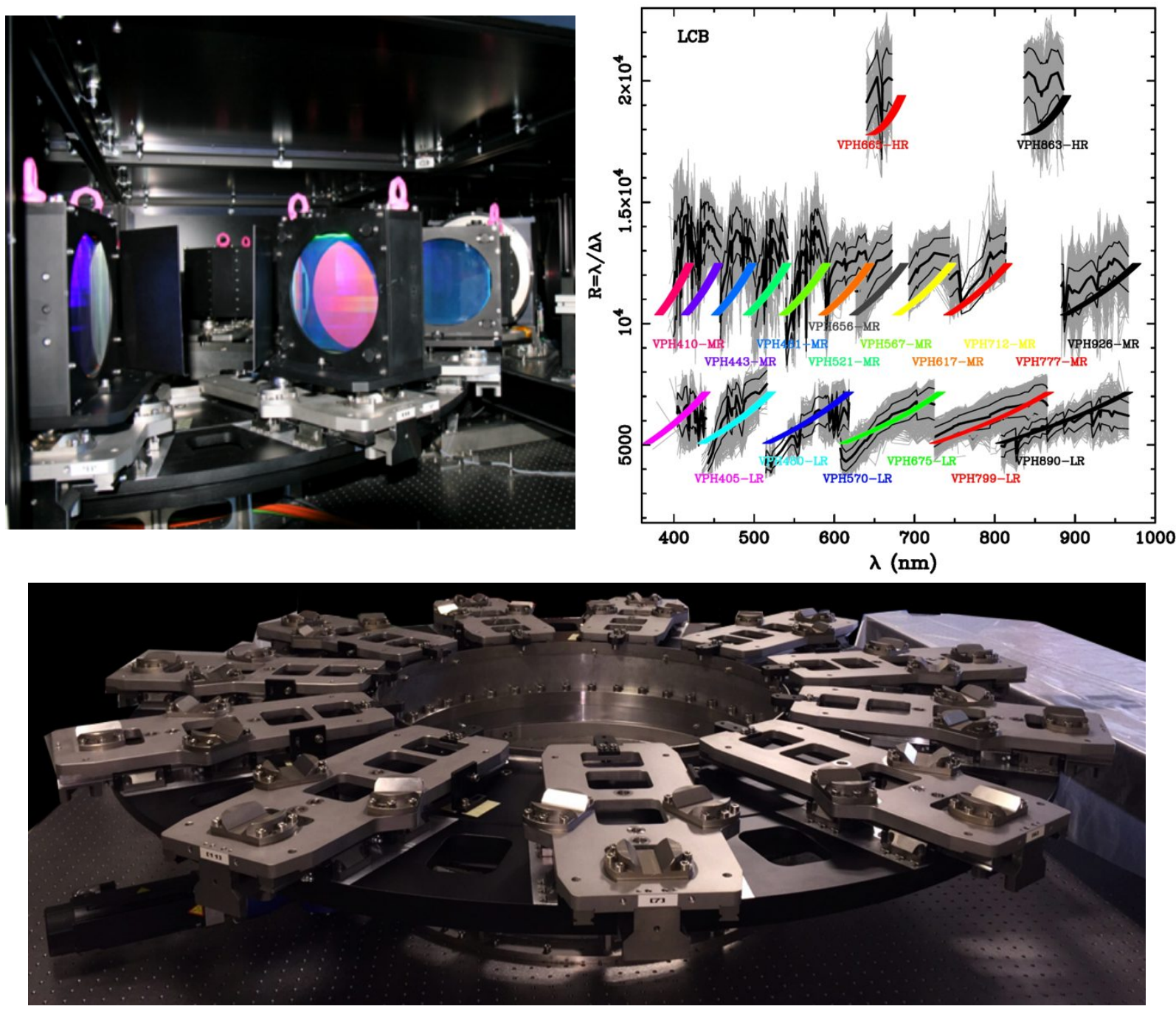

Figure 10: Top left: Inside view of the MEGARA spectrograph showing some VPHs mounted on the VPH wheel. All three VPHs shown at the front are LR VPHs, while a MR VPH can be seen in the background. Top right: Plot showing the relation between resolving power $\left(\mathrm{R}_{\mathrm{FWHM}}\right)$ and wavelength coverage for all 18 MEGARA VPHs. Shown in the plot are both design values (colored lines) and measurements (grey lines correspond to individual fiber spectra, while black thick and thin lines represent the mean and mean $\pm 1 \sigma$ curves when all fiber spectra are used). Bottom: Image of the VPH wheel before its integration with the MEGARA spectrograph.

The VPH wheel can host 11 VPHs of the total of 18 VPHs available for MEGARA (see Figure 10). Each of these 11 VPHs can be exchanged at the pupil position in less than one minute thanks to the combination of wheel rotation and insertion linear motion. The insertion of the VPH in the pupil is done by means of a linear stage with a travel distance from the wheel to the pupil position of $195 \mathrm{~mm}$ and the use of a series of interlock signals from multiple limit switches 
(see Section 3.5 below for a description of the MEGARA Control System including the control hardware, software and interlocks control).

\subsection{Control system}

The MEGARA Control System (MCS) is in charge of handling all the MEGARA mechanisms, the Data Acquisition System (DAS), the Inspector panels and sequencer strategies for observation. Besides, we have also developed commonuser software to be used by the GTC support astronomers and by the GTC astronomical community at large that constitutes the MEGARA Observing Preparation Software Suite (MOPSS) and Data Reduction Pipeline (DRP).

In this section we will briefly describe the different parts of the hardware and software that provide all these capabilities. All MCS components are compliant with the GTC software and hardware standards. In Figure 11 we show a graphical summary of the Control hardware (left) and the routing (right) of the Control hardware to the mechanisms, sensors and interlocks that are placed at the MEGARA optical bench in the Nasmyth A platform and at the Fiber-MOS at FC-F.
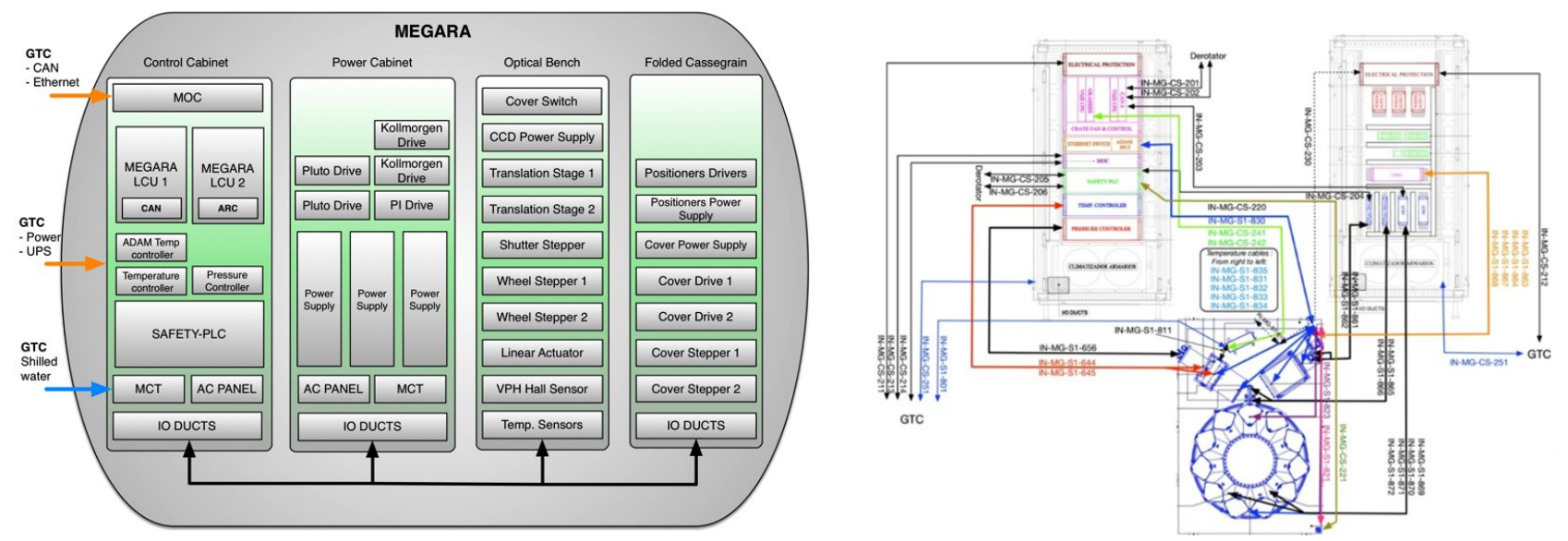

Figure 11. Left: MEGARA Control System hardware overview. Note that all this hardware is physically distributed in two cabinets placed at the Nasmyth A platform of GTC (the so-called Control and Power cabinet) and in the electronic boxes that are part of the Folded Cassegrain rotator adapter (see Figure 4). Right: Routing of the different hardware components of the MEGARA instrument.

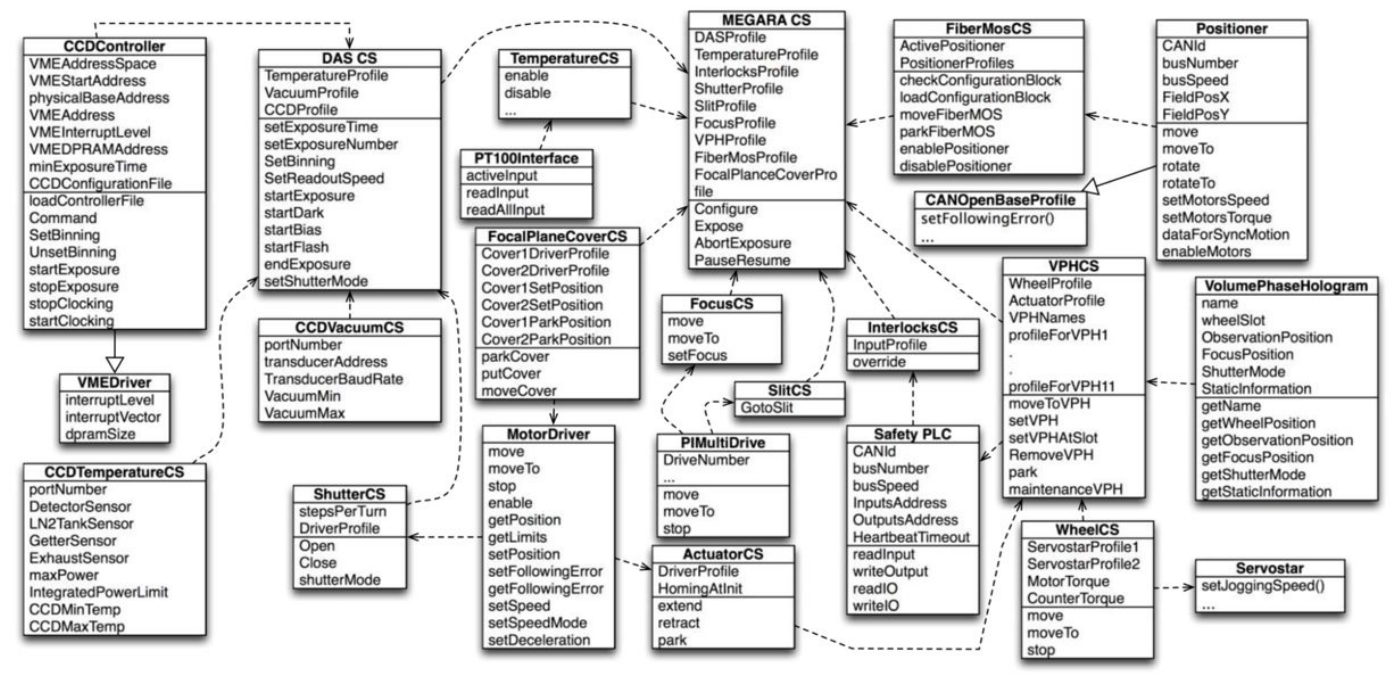

Figure 12: MEGARA Control System software classes. 
Regarding the Control Software, the MCS is constituted by a total of nine devices that include those that handle the mechanisms (pseudo-slit, focus, shutter, VPH wheel, Fiber-MOS, focal-plane cover), the DAS, sensors and interlocks. Figure 12 shows the main classes involved in MEGARA Control System.

The MCS also includes all GTC inspector panels needed to operate the instrument. In Figure 13 we show the main inspector panel of the MCS, which includes the visualization of the raw data (top left), the configuration of the VPH wheel, the cover and the pseudo-slit mechanisms (top right), the observing mode (bottom left) and the DAS and Instrument Calibration Module (ICM) (bottom right).

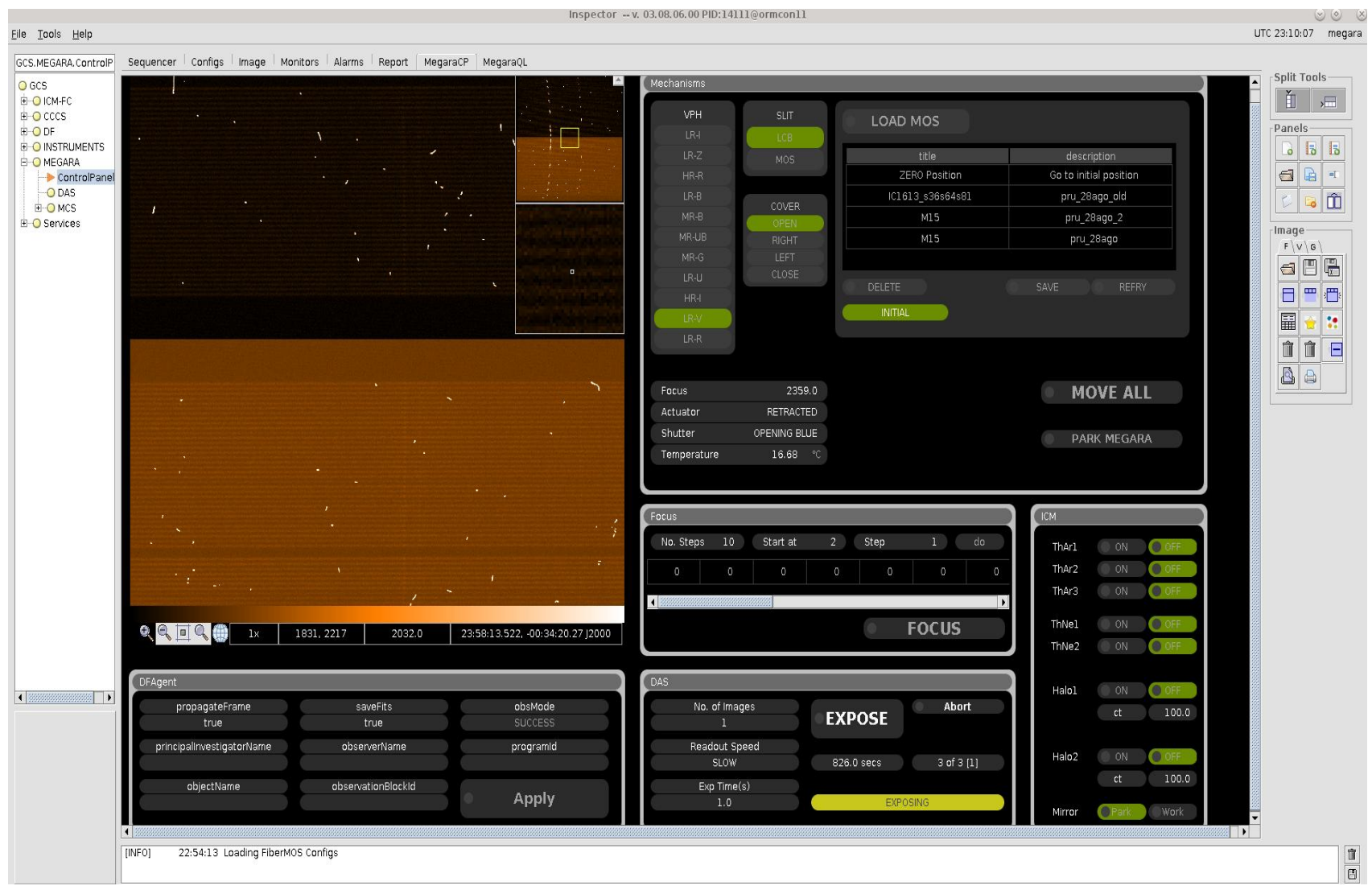

Figure 13: MCS panel of the GTC inspector. This panel is used for carrying out the observations with MEGARA at GTC.

Besides, the software developed as part of the MEGARA project includes the MOPSS, a suite of software tools designed for preparing and optimizing the observations with MEGARA, and the DRP. The software included in the MOPSS are the Exposure Time Calculator (ETC, http://gtc-phase2.gtc.iac.es/mect/etc/), the Fiber-MOS Assignment Tool (FMAT, http://www.gtc.iac.es/instruments/megara/config/megara-fmat.zip), and the MEGARA quick-look, which is embedded into the GTC inspector (see Figure 14). The MEGARA DRP is a python package that is able (1) to do the online processing of the MEGARA observations at GTC, including bias correction, trimming, fiber tracing and fixed-aperture extraction, fiber-flat and twilight-flat correction and wavelength calibration and (2) to do the offline processing, which also includes a detailed cross-talk corrected extraction and the flux calibration. The MEGARA DRP is distributed by GTC at http://www.gtc.iac.es/instruments/megara/config/megaradrp-0.6.dev2.tar.gz. Updates to the DRP can be obtained through github at https://github.com/guaix-ucm/megaradrp. 


\section{INTEGRATION AND COMMISSIONING RESULTS}

In this section we describe the main results of the integration and commissioning of MEGARA at GTC. Section 4.1 is devoted to the integration phase, while section 4.2 describes the daytime commissioning and section 4.3 the night-time commissioning phase.

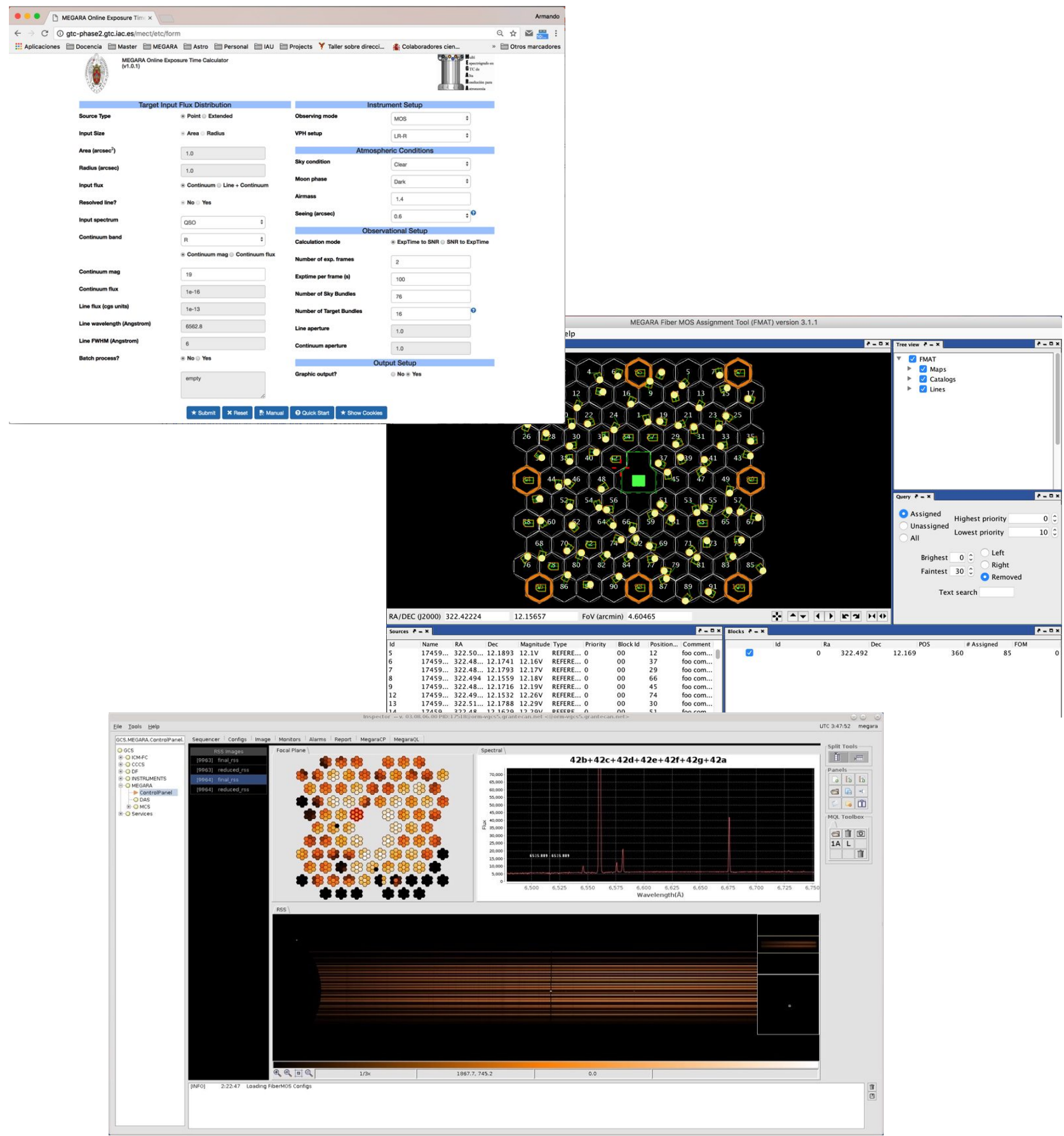

Figure 14. Top: Screen capture of the web-based MEGARA Exposure Time Calculator (ETC). Center: Screen capture of the Java stand-alone Fiber-MOS Assignment Tool (FMAT). Bottom: Screen capture of the quick-look shown integrated in the GTC inspector. 


\subsection{Integration}

The MEGARA integration plan at the telescope started at the beginning of the construction phase. MEGARA integration tasks started after unpacking the instrument at GTC and finished right after the team verified that the instrument was ready for starting the daytime commissioning (see Section 4.2). As part of the integration plan we also identified the resources (manpower, equipment and supplies) needed at GTC for carrying out a safe, timely and successful integration of the instrument. More information on the tasks included in the integration plan, the resources needed from the team and from GTC and the actual integration calendar are all described in detail in the accompanying paper by Pérez-Calpena et al. $(2018)^{17}$.

We foresaw that the most critical task was the integration of the FC assembly and the fiber bundles so we carried out two visits to GTC in advance to the actual integration (on June 2013 and July 2016) to simulate this procedure. The time estimates obtained from these visits and for the rest of the talks planned were considered together with GTC constrains in order to prepare a detailed integration calendar that was discussed and agreed with the Head of GTC Operations. The integration was carried out at daytime during three different periods at GTC: April $3^{\text {rd }}-$ April $7^{\text {th }} 2017$, April $17^{\text {th }}-21^{\text {st }}$ 2017 and April $24^{\text {th }}-28^{\text {th }} 2017$. The preparatory work, mainly outside the telescope chamber, such as the unpacking and testing of the electronic cabinets cooling circuits was done on March $29^{\text {th }}-31^{\text {st }} 2017$. The other three integration periods carried out on April 2017 were used to perform the integration tasks define in the integration plan.

\subsection{Daytime commissioning}

The commissioning plan was agreed with GTC at the time of the instrument Laboratory Acceptance. This commissioning plan included both daytime and night-time commissioning observing periods. As part of the former we defined a series of tasks that were focused mainly on the characterization of the different 18 spectral setups in the two instrument modes, the LCB IFU and the MOS. In Figure 15 we show the distribution of observations taken as part of the daytime commissioning periods in each mode and VPH setup. These daytime commissioning observations were taken as part of two runs that took place between May $8^{\text {th }}-12^{\text {th }} 2017$ and June $5^{\text {th }}-9^{\text {th }} 2017$.

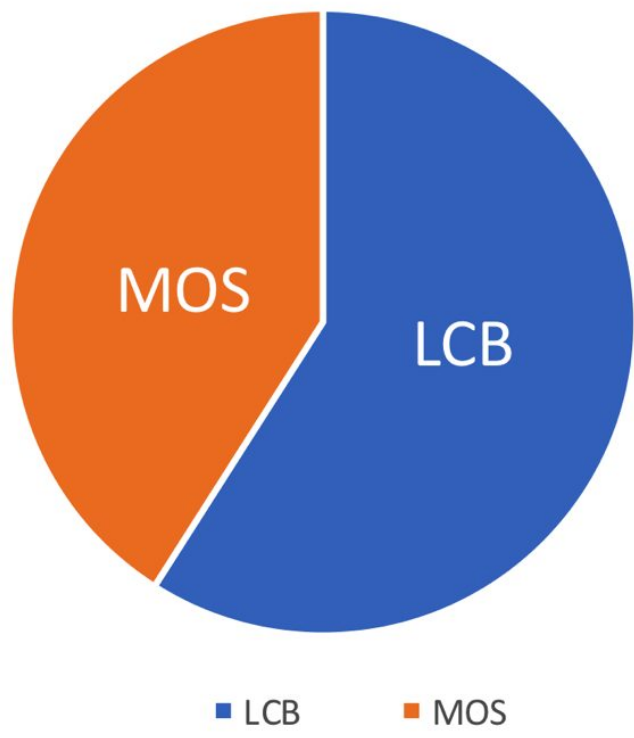

Figure 15: Left: Distribution of images taken in LCB (497 images) and MOS (345 images) modes as part of the daytime commissioning observations. Right: Distribution by spectral setup in all LR (447 images), MR (298 images), and HR (63) VPHs. The largest blue section in the distribution corresponds to the 248 LR-I VPH images obtained to perform the tests that were not spectral resolution dependent as mechanisms repeatibility, amongst others. 
During the daytime commissioning observations we obtained the optimal focus position for each VPH as a function of the mode (LCB or MOS) and temperature, although only two temperature values could be explored in all VPHs, T=11 ${ }^{\circ}$ and $21^{\circ}$, both very high values within the nominal operating temperature range of GTC (between $-2^{\circ}$ and $+19^{\circ}$ ). These optimal focus values were obtained by acquiring ThAr or ThNe arc lamp images at different focus positions and then measuring the FWHM of the extracted spectra averaged over all lines identified by the MEGARA DRP. In Figure 16 we show the optimal-focus curves obtained for all VPHs (each VPH is represented by its central wavelength) at the two temperatures quoted above along with the best-fitting polynomial for each temperature. The corresponding look-up table for setting up the focus for each VPH as a function of temperature is already included as part of the MCS and it is set automatically every time any MEGARA mechanism is moved.

As part of the daytime commissioning we also determined the central wavelength, spectral coverage and resolution of all 36 spectral setups (see Table 2 and Figure 10). The values were obtained by taking a ThAr or ThNe arc lamp image at the optimal focus of each VPH and then using the corresponding wavelength-calibration solutions and optimal FWHM measurements as a function of wavelength. Mechanism repeatability and fiber-to-fiber homogeneity requirements were also verified as part of the observations carried out during the two daytime commissioning observing runs.

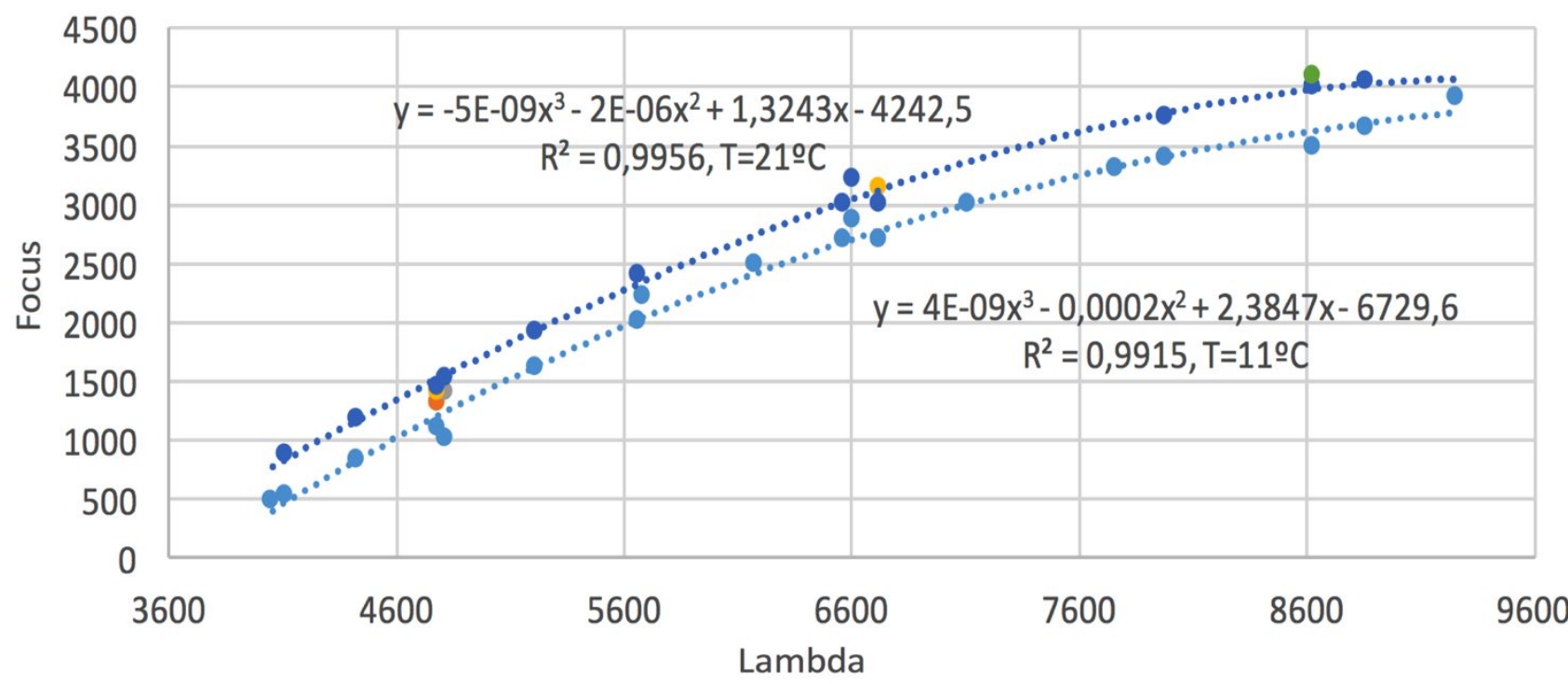

Figure 16: Focus curves obtained for different MEGARA VPHs at two different operating temperatures $\left(\mathrm{T}=11^{\circ}\right.$ and $\left.21^{\circ}\right)$. Best-fitting polynomial are also shown.

\subsection{Night-time commissioning}

The night-time commissioning tasks were defined and agreed with GTC along with the daytime ones. The success of the daytime observations allowed us to move forward to the first night-time commissioning run that was held during the nights between June $24^{\text {th }}$ and July $3^{\text {rd }} 2017$. The other two night-time runs of the commissioning took place between July $24^{\text {th }}$ and August $2^{\text {nd }} 2017$ and between August $22^{\text {nd }}$ and August $31^{\text {st }}$ 2017. The first light (on-sky) image of MEGARA was taken on June $24^{\text {th }} 2017$ with the observation of the spectrophotometric standard star and pre-planetary nebula ${ }^{18}$ $\mathrm{BD}+33^{\circ} 2642$. A detailed description of the astronomical targets observed as part of these commissioning observations are described in the accompanying contribution by Gil de Paz et al. $(2018)^{6}$. This paper provides also more details on the rest of the night-time commissioning observations, which also includes the observation of multiple calibration images, such as twilight and ICM flats, arc lamps or focus sequences (see Figure 13).

As shown in Figure 17, a total of 2570 LCB (for a total of 1,598,540 individual fiber spectra) and 847 MOS (544,621 spectra) were taken during the three commissioning runs. These observations allowed us to carry out the Fiber-MOS dynamic tilt assessment, to determine a precise value for the Instrument Alignment Angle, and to estimate the positioning errors for each of the MOS robotic positioners along with the efficiency of the instrument in both modes and in all 18 VPHs. 


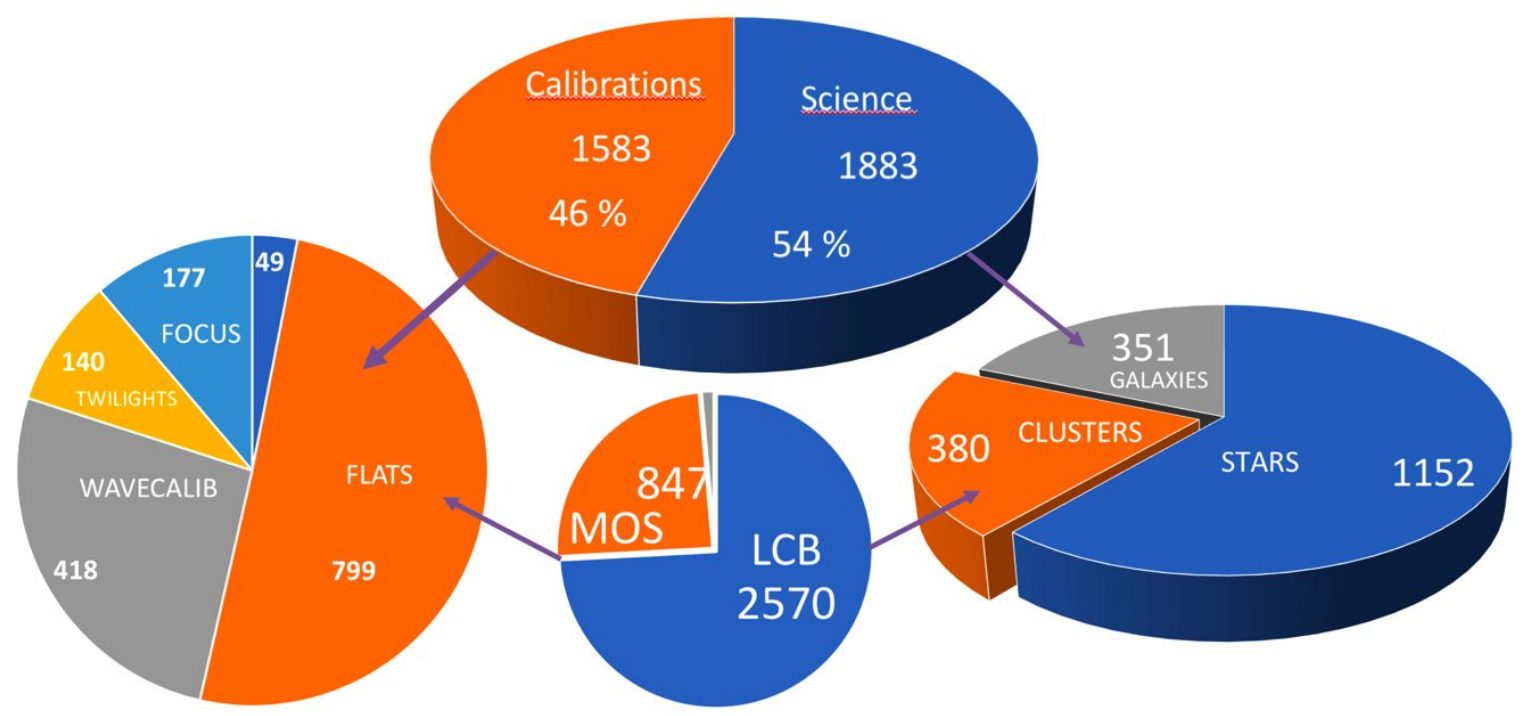

Figure 17: Distribution by image type, observing mode, instrument mode and object type of the night-time commissioning images.

\section{CONCLUSIONS}

MEGARA is a case of success, that is, the design, construction, integration and commissioning were carried out fulfilling all the requirements, timely and within budget. The construction contract was signed by GRANTECAN and UCM in May 5, 2014. MEGARA arrived to the observatory in March 28, 2017. The integration and daytime and night-time commissioning at GTC ended in August 31, 2017, in a record time of over 3 years after the contract signature. MEGARA is now a facility instrument that has become available to the GTC community.

\section{REFERENCES}

[1] Gil de Paz, A., "MEGARA: the future optical IFU and multi-object spectrograph for the $10.4 \mathrm{~m}$ GTC telescope", Proc. SPIE Vol. 8446, 84464Q (2012)

[2] Gil de Paz, A., et al., "MEGARA: The future IFU \& MOS of the 10.4m GTC", Revista Mexicana de Astronomía y Astrofísica (Serie de Conferencias) 42, 90-92 (2013)

[3] Gil de Paz, A., et al., "MEGARA: a new generation optical spectrograph for GTC", Proc. SPIE, Vol. 9147, id. 914700 (2014)

[4] Gil de Paz, A., et al., "MEGARA, the new IFU and MOS for the GTC", Highlights of Spanish Astrophysics VIII, Proc. of the XI SEA Scientific Meeting. A. J. Cenarro et al. (eds.), p. 804 (2015)

[5] Gil de Paz, A., et al., "MEGARA, the new intermediate-resolution optical IFU and MOS for GTC: getting ready for the telescope", Proc. SPIE Vol. 9908, 99081K (2016)

[6] Gil de Paz, A., et al., "First scientific observations with MEGARA at GTC", Proc. of SPIE 10702, paper: 10702-43 (2018).

[7] Sheth, K., et al., "The Spitzer Survey of Stellar Structure in Galaxies $\left(\mathrm{S}^{4} \mathrm{G}\right)$ ", Publications of the Astronomical Society of the Pacific 122, 1397-1414 (2010) 
[8] Mayya, Y.D. et al., "The SFH of the Disk of the Starburst M82", The Astrophysical Journal, 649, 172 (2006)

[9] Goudfrooij, P. et al., "Kinematics, ages and metallicities of star clusters in NGC 1316: a 3-Gyr-old merger remnant", Monthly Notices of the Royal Astronomical Society, 322, 643 (2001)

[10] Peimbert M., Storey P. J., Torres-Peimbert S., "The $\mathrm{O}(2+) / \mathrm{H}(+)$ abundance ratio in gaseous nebulae derived from recombination lines", The Astrophysical Journal, 414, 626 (1993)

[11]Liu X.-W., Storey P. J., Barlow M. J., Clegg R. E. S., "The rich O II recombination spectrum of the PN NGC 7009: new observations and atomic data", Monthly Notices of the Royal Astronomical Society, 272, 369 (1995)

[12] Jones D., Wesson R., García-Rojas J., Corradi R. L. M., Boffin H. M. J., "NGC 6778: strengthening the link between extreme abundance discrepancy factors and central star binarity in planetary nebulae", Monthly Notices of the Royal Astronomical Society, 455, 3263 (2016)

[13] Kharchenko, N.V., et al., "Global survey of star clusters in the Milky Way. II. The catalogue of basic parameters", Astronomy \& Astrophysics, 558, A53 (2013)

[14] Amorín, R., et al., "Extreme emission-line galaxies out to $\mathrm{z} \sim 1$ in zCOSMOS. I. Sample and characterization of global properties", Astronomy \& Astrophysics, 578A, 105 (2015)

[15] Murphy, T.W., Jr. et al., "Visual and Near-Infrared Imaging of Ultraluminous Infrared Galaxies: The IRAS 2 Jy Sample", Astronomical Journal, 111, 1025 (1996)

[16] Pérez-Calpena, A., et al., " MEGARA MOS: where are my positions and fibres pointing to?", Proc. of SPIE 10706, paper: 10706- 82 (2018).

[17] Pérez-Calpena, A., et al., "Delivery and integration of MEGARA at GTC: the process of going from laboratory to the telescope". Proc of SPIE 10705, paper: 10705-13 (2018).

[18] Napiwotzki, R., "BD+33²642 - a new planetary nebula in the Galactic halo", Acta Astron., 43, 415 (1993) 